\title{
Arens regularity of module actions
}

\author{
by \\ M. Eshaghi Gordji (Semnan and Tehran) and M. Filali (Oulu)
}

\begin{abstract}
We study the Arens regularity of module actions of Banach left or right modules over Banach algebras. We prove that if $\mathcal{A}$ has a brai (blai), then the right (left) module action of $\mathcal{A}$ on $\mathcal{A}^{*}$ is Arens regular if and only if $\mathcal{A}$ is reflexive. We find that Arens regularity is implied by the factorization of $\mathcal{A}^{*}$ or $\mathcal{A}^{* *}$ when $\mathcal{A}$ is a left or a right ideal in $\mathcal{A}^{* *}$. The Arens regularity and strong irregularity of $\mathcal{A}$ are related to those of the module actions of $\mathcal{A}$ on the $n$th dual $\mathcal{A}^{(n)}$ of $\mathcal{A}$. Banach algebras $\mathcal{A}$ for which $Z\left(\mathcal{A}^{* *}\right)=\mathcal{A}$ but $\mathcal{A} \subsetneq Z^{t}\left(\mathcal{A}^{* *}\right)$ are found (here $Z\left(\mathcal{A}^{* *}\right)$ and $Z^{t}\left(\mathcal{A}^{* *}\right)$ are the topological centres of $\mathcal{A}^{* *}$ with respect to the first and second Arens product, respectively). This also gives examples of Banach algebras such that $\mathcal{A} \subsetneq Z\left(\mathcal{A}^{* *}\right) \subsetneq \mathcal{A}^{* *}$. Finally, the triangular Banach algebras $\mathcal{T}$ are used to find Banach algebras having the following properties: (i) $\mathcal{T}^{*} \mathcal{T}=\mathcal{T}^{*}$ but $Z\left(\mathcal{T}^{* *}\right) \neq Z^{t}\left(\mathcal{T}^{* *}\right)$; (ii) $Z\left(\mathcal{T}^{* *}\right)=Z^{t}\left(\mathcal{T}^{* *}\right)$ and $\mathcal{T}^{*} \mathcal{T}=\mathcal{T}^{*}$ but $\mathcal{T}^{*} \neq \mathcal{T}^{*}$; (iii) $Z\left(\mathcal{T}^{* *}\right)=\mathcal{T}$ but $\mathcal{T}$ is not weakly sequentially complete. The results (ii) and (iii) are new examples answering questions asked by Lau and Ülger.
\end{abstract}

1. Introduction. The extension of bilinear maps on normed spaces and the concept of regularity of bilinear maps were introduced by Richard Arens in 1951 (see [1] and [2]). We start by recalling these definitions. Throughout the paper, we shall identify any Banach space with its natural image in the second dual. Let $X, Y$ and $Z$ be normed spaces and let $f: X \times Y \rightarrow Z$ be a continuous bilinear map. Then the adjoint of $f$ is defined by

$$
f^{*}: Z^{*} \times X \rightarrow Y^{*}, \quad\left\langle f^{*}\left(z^{\prime}, x\right), y\right\rangle=\left\langle z^{\prime}, f(x, y)\right\rangle
$$

$$
\left(z^{\prime} \in Z^{*}, x \in X, y \in Y\right) \text {. }
$$

Clearly, for each $x \in X$, the map $z^{\prime} \mapsto f^{*}\left(z^{\prime}, x\right): Z^{*} \rightarrow Y^{*}$ is weak*weak* continuous. Since $f^{*}$ is a continuous bilinear map, this process may be repeated to define $f^{* *}=\left(f^{*}\right)^{*}: Y^{* *} \times Z^{*} \rightarrow X^{*}$, and then $f^{* * *}=\left(f^{* *}\right)^{*}$ : $X^{* *} \times Y^{* *} \rightarrow Z^{* *}$. The map $f^{* * *}$ is the unique extension of $f$ such that

- $x^{\prime \prime} \mapsto f^{* * *}\left(x^{\prime \prime}, y^{\prime \prime}\right): X^{* *} \rightarrow Z^{* *}$ is weak*-weak* continuous for each $y^{\prime \prime} \in Y^{* *}$,

2000 Mathematics Subject Classification: Primary 46H25; Secondary 46H20, 43A20.

Key words and phrases: Arens product, topological centres, strongly Arens irregular, module actions. 
- $y^{\prime \prime} \mapsto f^{* * *}\left(x, y^{\prime \prime}\right): Y^{* *} \rightarrow Z^{* *}$ is weak $^{*}$-weak ${ }^{*}$ continuous for each $x \in X$.

The first topological centre of $f$ may therefore be defined as

$$
\begin{aligned}
Z(f)=\left\{x^{\prime \prime} \in X^{* *}: y^{\prime \prime} \mapsto f^{* * *}\left(x^{\prime \prime}, y^{\prime \prime}\right): Y^{* *}\right. & \rightarrow Z^{* *} \text { is } \\
& \text { weak } \left.^{*} \text {-weak }^{*} \text { continuous }\right\} .
\end{aligned}
$$

Let now $f^{t}: Y \times X \rightarrow Z$ be the transpose of $f$ defined by $f^{t}(y, x)=f(x, y)$ for all $x \in X$ and $y \in Y$. Then $f^{t}$ is a continuous bilinear map from $Y \times X$ to $Z$, and so it may be extended as above to $f^{t * * *}: Y^{* *} \times X^{* *} \rightarrow Z^{* *}$. Consider next the map $f^{t * * * t}: X^{* *} \times Y^{* *} \rightarrow Z^{* *}$. Again we have:

- $y^{\prime \prime} \mapsto f^{t * * * t}\left(x^{\prime \prime}, y^{\prime \prime}\right): Y^{* *} \rightarrow Z^{* *}$ is weak $^{*}$-weak* continuous for each $x^{\prime \prime} \in X^{* *}$

- $x^{\prime \prime} \mapsto f^{t * * * t}\left(x^{\prime \prime}, y\right): X^{* *} \rightarrow Z^{* *}$ is weak ${ }^{*}$ weak $^{*}$ continuous for each $y \in Y$.

The second topological centre of $f$ may be defined as

$$
\begin{aligned}
& Z^{t}(f)=\left\{y^{\prime \prime} \in Y^{* *}: x^{\prime \prime} \mapsto f^{t * * *}\left(y^{\prime \prime}, x^{\prime \prime}\right): X^{* *} \rightarrow Z^{* *}\right. \text { is } \\
&\text { weak } \left.^{*} \text {-weak }^{*} \text { continuous }\right\} \\
&=\left\{y^{\prime \prime} \in Y^{* *}: x^{\prime \prime} \mapsto f^{t * * * t}\left(x^{\prime \prime}, y^{\prime \prime}\right): X^{* *} \rightarrow Z^{* *}\right. \text { is } \\
&\text { weak } \left.^{*} \text {-weak }^{*} \text { continuous }\right\} .
\end{aligned}
$$

Clearly, $X \subseteq Z(f)$ and $Y \subseteq Z^{t}(f)$.

The map $f$ is Arens regular when $f^{* * *}=f^{t * * * t}$. This is equivalent to the condition that the map

$$
y^{\prime \prime} \mapsto f^{* * *}\left(x^{\prime \prime}, y^{\prime \prime}\right): Y^{* *} \rightarrow Z^{* *}
$$

is weak*-weak* continuous for every $x^{\prime \prime} \in X^{* *}$, i.e., $Z(f)=X^{* *}$; and to the condition that the map

$$
x^{\prime \prime} \mapsto f^{t * * * t}\left(x^{\prime \prime}, y^{\prime \prime}\right): X^{* *} \rightarrow Z^{* *}
$$

is weak ${ }^{*}$-weak ${ }^{*}$ continuous for every $y^{\prime \prime} \in Y^{* *}$, i.e., $Z\left(f^{t}\right)=Y^{* *}$.

The map $f$ is left strongly Arens irregular when $Z(f)=X$, right strongly Arens irregular when $Z\left(f^{t}\right)=Y$, and strongly Arens irregular when $Z(f)=X$ and $Z\left(f^{t}\right)=Y$.

Let now $X$ be a Banach $\mathcal{A}$-bimodule, and let

$$
\pi_{l}: \mathcal{A} \times X \rightarrow X \quad \text { and } \quad \pi_{r}: X \times \mathcal{A} \rightarrow X
$$

be the right and left module actions of $\mathcal{A}$ on $X$. Then $X^{* *}$ is a Banach $\mathcal{A}^{* *}$-bimodule with module actions

$$
\pi_{l}^{* * *}: \mathcal{A}^{* *} \times X^{* *} \rightarrow X^{* *} \text { and } \pi_{r}^{* * *}: X^{* *} \times \mathcal{A}^{* *} \rightarrow X^{* *} .
$$

Similarly, $X^{* *}$ is a Banach $\mathcal{A}^{* *}$-bimodule with module actions

$$
\pi_{l}^{t * * * t}: \mathcal{A}^{* *} \times X^{* *} \rightarrow X^{* *} \text { and } \pi_{r}^{t * * * t}: X^{* *} \times \mathcal{A}^{* *} \rightarrow X^{* *} .
$$


We may therefore define the topological centres of the right and left module actions of $\mathcal{A}$ on $X$ as follows:

$$
\begin{aligned}
& Z_{\mathcal{A}}\left(X^{* *}\right):=Z\left(\pi_{r}\right)=\left\{x^{\prime \prime} \in X^{* *}: \text { the map } a^{\prime \prime} \mapsto \pi_{r}^{* * *}\left(x^{\prime \prime}, a^{\prime \prime}\right):\right. \\
& \left.\mathcal{A}^{* *} \rightarrow X^{* *} \text { is weak*-weak }{ }^{*} \text { continuous }\right\} \text {, } \\
& Z_{X}\left(\mathcal{A}^{* *}\right):=Z\left(\pi_{l}\right)=\left\{a^{\prime \prime} \in \mathcal{A}^{* *}: \text { the map } x^{\prime \prime} \mapsto \pi_{l}^{* * *}\left(a^{\prime \prime}, x^{\prime \prime}\right)\right. \text { : } \\
& \left.X^{* *} \rightarrow X^{* *} \text { is weak*-weak }{ }^{*} \text { continuous }\right\}, \\
& Z_{\mathcal{A}}^{t}\left(X^{* *}\right):=Z\left(\pi_{l}^{t}\right)=\left\{x^{\prime \prime} \in X^{* *}: \text { the map } a^{\prime \prime} \mapsto \pi_{l}^{t * * *}\left(x^{\prime \prime}, a^{\prime \prime}\right)\right. \text { : } \\
& \left.\mathcal{A}^{* *} \rightarrow X^{* *} \text { is weak*-weak }{ }^{*} \text { continuous }\right\} \text {, } \\
& Z_{X}^{t}\left(\mathcal{A}^{* *}\right):=Z\left(\pi_{r}^{t}\right)=\left\{a^{\prime \prime} \in \mathcal{A}^{* *}: \text { the map } x^{\prime \prime} \mapsto \pi_{r}^{t * * *}\left(a^{\prime \prime}, x^{\prime \prime}\right):\right. \\
& \left.X^{* *} \rightarrow X^{* *} \text { is weak*-weak }{ }^{*} \text { continuous }\right\} \text {. }
\end{aligned}
$$

We note also that if $X$ is a left (right) Banach $\mathcal{A}$-module and $\pi_{l}: \mathcal{A} \times X$ $\rightarrow X\left(\pi_{r}: X \times \mathcal{A} \rightarrow X\right)$ is the left (right) module action of $\mathcal{A}$ on $X$, then $X^{*}$ is a right (left) Banach $\mathcal{A}$-module. The right module action is given by $\pi_{l}^{*}: X^{*} \times \mathcal{A} \rightarrow X^{*}$, where as above,

$$
\left\langle\pi_{l}^{*}\left(x^{\prime}, a\right), x\right\rangle=\left\langle x^{\prime}, \pi_{l}(a, x)\right\rangle \quad\left(a \in \mathcal{A}, x^{\prime} \in X^{*}, x \in X\right),
$$

which we shall write simply as $\left\langle x^{\prime} a, x\right\rangle=\left\langle x^{\prime}, a x\right\rangle$ when there is no confusion. The left module action is given by $\pi_{r}^{t * t}: \mathcal{A} \times X^{*} \rightarrow X^{*}$, where as above,

$$
\left\langle\pi_{r}^{t * t}\left(a, x^{\prime}\right), x\right\rangle=\left\langle\pi_{r}^{t *}\left(x^{\prime}, a\right), x\right\rangle=\left\langle x^{\prime}, \pi_{r}^{t}(a, x)\right\rangle=\left\langle x^{\prime}, \pi_{r}(x, a)\right\rangle
$$

for all $a \in \mathcal{A}, x^{\prime} \in X^{*}, x \in X$, which we shall also write as $\left\langle a x^{\prime}, x\right\rangle=\left\langle x^{\prime}, x a\right\rangle$ when no confusion can arise.

Similarly, $X^{* *}, \ldots, X^{(n)}$ are left or right Banach $\mathcal{A}$-modules. Therefore we may start with the operation $\pi: \mathcal{A} \times \mathcal{A} \rightarrow \mathcal{A}$ and consider $\mathcal{A}^{*}, \mathcal{A}^{* *}, \ldots, \mathcal{A}^{(n)}$ as $\mathcal{A}$-bimodules for each $n \geq 1$. We denote by $\pi_{r_{n}}: \mathcal{A}^{(n)} \times \mathcal{A} \rightarrow \mathcal{A}^{(n)}$ and $\pi_{l_{n}}: \mathcal{A} \times \mathcal{A}^{(n)} \rightarrow \mathcal{A}^{(n)}$, respectively, the right and left module actions of $\mathcal{A}$ on $\mathcal{A}^{(n)}$. Here $\pi_{l_{n}}=\pi_{r_{n-1}}^{t * t}$ and $\pi_{r_{n}}=\pi_{l_{n-1}}^{*}$ for all $n \geq 1$ with $\mathcal{A}^{(0)}=\mathcal{A}$ and $\pi_{l_{0}}=\pi_{r_{0}}=\pi$.

Regarding $\mathcal{A}$ as a Banach $\mathcal{A}$-bimodule, the operation $\pi: \mathcal{A} \times \mathcal{A} \rightarrow \mathcal{A}$ extends to $\pi^{* * *}$ and $\pi^{t * * * t}$ defined on $\mathcal{A}^{* *} \times \mathcal{A}^{* *}$. These extensions are known, respectively, as the first (left) and the second (right) Arens products, and with each of them, the second dual space $\mathcal{A}^{* *}$ becomes a Banach algebra. In this situation, we shall also simplify our notations. So the first (left) Arens product of $a^{\prime \prime}, b^{\prime \prime} \in \mathcal{A}^{* *}$ will be simply indicated by $a^{\prime \prime} b^{\prime \prime}$ and defined by the following three steps:

$$
\left\langle a^{\prime} a, b\right\rangle=\left\langle a^{\prime}, a b\right\rangle,\left\langle b^{\prime \prime} a^{\prime}, a\right\rangle=\left\langle b^{\prime \prime}, a^{\prime} a\right\rangle,\left\langle a^{\prime \prime} b^{\prime \prime}, a^{\prime}\right\rangle=\left\langle a^{\prime \prime}, b^{\prime \prime} a^{\prime}\right\rangle
$$

for all $a^{\prime} \in \mathcal{A}^{*}, a, b \in \mathcal{A}$. Similarly, the second (right) Arens product of $a^{\prime \prime}, b^{\prime \prime} \in \mathcal{A}^{* *}$ will be indicated by $a^{\prime \prime} . b^{\prime \prime}$ and defined by 


$$
\left\langle a \cdot a^{\prime}, b\right\rangle=\left\langle a^{\prime}, b a\right\rangle,\left\langle a^{\prime} \cdot a^{\prime \prime}, a\right\rangle=\left\langle a^{\prime \prime}, a \cdot a^{\prime}\right\rangle,\left\langle a^{\prime \prime} \cdot b^{\prime \prime}, a^{\prime}\right\rangle=\left\langle b^{\prime \prime}, a^{\prime} \cdot a^{\prime \prime}\right\rangle
$$

for all $a^{\prime} \in \mathcal{A}^{*}, a, b \in \mathcal{A}$.

We find the usual first and second topological centres of $\mathcal{A}^{* *}$, which are

$$
\begin{aligned}
Z\left(\mathcal{A}^{* *}\right) & =\left\{a^{\prime \prime} \in \mathcal{A}^{* *}: b^{\prime \prime} \mapsto a^{\prime \prime} b^{\prime \prime} \text { is weak }{ }^{*} \text {-weak }{ }^{*} \text { continuous }\right\} \\
Z^{t}\left(\mathcal{A}^{* *}\right) & =\left\{b^{\prime \prime} \in \mathcal{A}^{* *}: a^{\prime \prime} \mapsto a^{\prime \prime} . b^{\prime \prime} \text { is weak*-weak }{ }^{*} \text { continuous }\right\}
\end{aligned}
$$

Following our previous notations, $Z\left(\mathcal{A}^{* *}\right)=Z(\pi)=Z_{\mathcal{A}}\left(\mathcal{A}^{* *}\right)$ and $Z^{t}\left(\mathcal{A}^{* *}\right)=$ $Z^{t}(\pi)=Z_{\mathcal{A}}^{t}\left(\mathcal{A}^{* *}\right)$.

A Banach algebra $\mathcal{A}$ is Arens regular when $Z\left(\mathcal{A}^{* *}\right)=\mathcal{A}^{* *}$, or equivalently, $Z^{t}\left(\mathcal{A}^{* *}\right)=\mathcal{A}^{* *}$; and according to [5], $\mathcal{A}$ is strongly Arens irregular when $Z\left(\mathcal{A}^{* *}\right)=Z^{t}\left(\mathcal{A}^{* *}\right)=\mathcal{A}$.

For more information, the reader is directed to [4], [5], [7], [10] and [17].

Recall that a bounded left approximate identity (blai) in a Banach algebra $\mathcal{A}$ is a bounded net $\left(e_{\alpha}\right)$ in $\mathcal{A}$ such that $\left\|e_{\alpha} a-a\right\| \rightarrow 0$. A bounded right approximate identity (brai) is defined similarly. A bounded approximate identity (bai) is a net $\left(e_{\alpha}\right)$ which is both a blai and a brai.

The paper is organized as follows. In Section 2, we prove that if $\mathcal{A}$ has a brai (blai), then the right (left) module action of $\mathcal{A}$ on $\mathcal{A}^{*}$ is Arens regular if and only if $\mathcal{A}$ is reflexive. This includes results proved by Arıkan in [3], Ülger in [19], and Dales, Rodríguez and Velasco in [6].

In Section 3, we see in particular that if $\mathcal{A}$ has a brai (or a blai) and the left module action of $\mathcal{A}$ on $\mathcal{A}^{(2 k)}$ (or $\mathcal{A}^{(2 k-1)}$ ) is Arens regular for some $k \geq 1$, then $\mathcal{A}$ is Arens regular. If $\mathcal{A}$ has a brai (or a blai) and is strongly left (right) irregular, then the left (right) module action of $\mathcal{A}$ on $\mathcal{A}^{(2 k)}$ (or $\left.\mathcal{A}^{(2 k-1)}\right)$ is strongly Arens irregular for every $k \geq 1$.

In Section 4, we prove that $\mathcal{A}$ is Arens regular if $\mathcal{A}^{*}$ factors and $\mathcal{A}$ is a left ideal in $\mathcal{A}^{* *}$. A similar conclusion is obtained when $\mathcal{A}^{* *}$ (as a Banach $\mathcal{A}$-bimodule) factors and $\mathcal{A}$ is a right ideal in $\mathcal{A}^{* *}$. We also relate the factorization of $\mathcal{A}^{* *}$ to the weak ${ }^{*}$ cluster points in $\mathcal{A}^{* *}$ of a bai of $\mathcal{A}$.

In Section 5, we retake an example used by Ghahramani et al. in [12], and we provide in Theorem 5.1 a class of Banach algebras which are left strongly Arens irregular but not right strongly Arens irregular. This class includes the recent example given by Dales and Lau in [5, Example 4.5]. Another type of example was also provided by Neufang in [16]. Theorem 5.1 enables us also to give simple examples of Banach algebras that are neither Arens regular nor left strongly Arens irregular. An earlier example of the latter type of algebras was given by Saghafi in [18].

In Section 6, we determine the topological centres of the second duals of the so-called triangular Banach algebras. The Arens regularity of these algebras has been studied by Forrest and Marcoux in [11]. We then use these centres to show the following assertions: 
(i) $\mathcal{A}^{*} \mathcal{A}=\mathcal{A A}^{*}$ is not sufficient for $Z\left(\mathcal{A}^{* *}\right)=Z^{t}\left(\mathcal{A}^{* *}\right)$;

(ii) $Z\left(\mathcal{A}^{* *}\right)=Z^{t}\left(\mathcal{A}^{* *}\right)$ and $\mathcal{A}^{*} \mathcal{A}=\mathcal{A}^{*}$ is not sufficient for $\mathcal{A} \mathcal{A}^{*}=\mathcal{A}^{*}$;

(iii) there exists a Banach algebra $\mathcal{A}$ that is strongly irregular but not weakly sequentially complete.

The results (ii) and (iii) are new examples answering the questions asked by Lau and Ülger in [14]. Examples different from ours have also been given in $[12]$.

2. Arens regularity of the module actions of $\mathcal{A}$ on $\mathcal{A}^{*}$. Arıkan proved in [3] that a unital Banach algebra $\mathcal{A}$ is reflexive if and only if every left module action of $\mathcal{A}$ is Arens regular. Ülger extended this theorem by proving in [19] that a unital Banach algebra $\mathcal{A}$ is reflexive if and only if the left module action of $\mathcal{A}$ on the dual $\mathcal{A}^{*}$ of $\mathcal{A}$ is Arens regular. Dales, Rodríguez and Velasco obtained in [6] the same result when $\mathcal{A}$ has just a bounded left approximate identity (blai) but assumed that $\mathcal{A}$ is Arens regular. With a short and simpler proof, and without the condition that $\mathcal{A}$ is Arens regular, our first theorem includes all these results.

Theorem 2.1. Let $\mathcal{A}$ be a Banach algebra, and let $\pi: \mathcal{A} \times \mathcal{A} \rightarrow \mathcal{A}$ be the product of $\mathcal{A}$.

(1) If $\mathcal{A}$ has a bounded right approximate identity (brai), then $\mathcal{A}$ is reflexive if and only if the right module action of $\mathcal{A}$ on $\mathcal{A}^{*}$ is Arens regular, or equivalently, $Z_{\mathcal{A}}\left(\mathcal{A}^{* * *}\right)=\mathcal{A}^{* * *}$.

(2) If $\mathcal{A}$ has a bounded left approximate identity (blai), then $\mathcal{A}$ is reflexive if and only if the left module action of $\mathcal{A}$ on $\mathcal{A}^{*}$ is Arens regular, or equivalently, $Z_{\mathcal{A}^{*}}\left(\mathcal{A}^{* *}\right)=\mathcal{A}^{* *}$.

Proof. If $\mathcal{A}$ is reflexive, then the right and left module actions of $\mathcal{A}$ on $\mathcal{A}^{*}$ are obviously Arens regular.

For the converse, suppose that the right module action $\pi^{*}: \mathcal{A}^{*} \times \mathcal{A} \rightarrow \mathcal{A}^{*}$ of $\mathcal{A}$ on $\mathcal{A}^{*}$ is Arens regular, let $a^{\prime \prime \prime} \in \mathcal{A}^{* * *}$ be arbitrary, and $\left(a_{\alpha}^{\prime \prime}\right)_{\alpha}$ be a net weak* converging to some $a^{\prime \prime}$ in $A^{* *}$. Then $\pi^{* * * *}\left(a^{\prime \prime \prime}, a_{\alpha}^{\prime \prime}\right) \stackrel{\text { weak }^{*}}{\longrightarrow} \pi^{* * * *}\left(a^{\prime \prime \prime}, a^{\prime \prime}\right)$ in $\mathcal{A}^{* * *}$ (since the right action of $\mathcal{A}$ on $\mathcal{A}^{*}$ is regular). Thus for every $b^{\prime \prime} \in \mathcal{A}^{* *}$, we have

$$
\begin{aligned}
\lim _{\alpha}\left\langle a^{\prime \prime \prime}, a_{\alpha}^{\prime \prime} b^{\prime \prime}\right\rangle & =\lim _{\alpha}\left\langle a^{\prime \prime \prime}, \pi^{* * *}\left(a_{\alpha}^{\prime \prime}, b^{\prime \prime}\right)\right\rangle=\lim _{\alpha}\left\langle\pi^{* * * *}\left(a^{\prime \prime \prime}, a_{\alpha}^{\prime \prime}\right), b^{\prime \prime}\right\rangle \\
& =\left\langle\pi^{* * * *}\left(a^{\prime \prime \prime}, a^{\prime \prime}\right), b^{\prime \prime}\right\rangle=\left\langle a^{\prime \prime \prime}, \pi^{* * *}\left(a^{\prime \prime}, b^{\prime \prime}\right)\right\rangle=\left\langle a^{\prime \prime \prime}, a^{\prime \prime} b^{\prime \prime}\right\rangle .
\end{aligned}
$$

Let $e^{\prime \prime}$ be a cluster point of the brai of $\mathcal{A}$ in $\mathcal{A}^{* *}$. Then $e^{\prime \prime}$ is a right identity in $\mathcal{A}^{* *}$ (with the first Arens product which coincides with $\pi^{* * *}$ ), and so $a_{\alpha}^{\prime \prime} e^{\prime \prime}=a_{\alpha}^{\prime \prime}$ for each $\alpha$ and $a^{\prime \prime} e^{\prime \prime}=a^{\prime \prime}$. Accordingly,

$$
\lim _{\alpha}\left\langle a^{\prime \prime \prime}, a_{\alpha}^{\prime \prime}\right\rangle=\lim _{\alpha}\left\langle a^{\prime \prime \prime}, a_{\alpha}^{\prime \prime} e^{\prime \prime}\right\rangle=\left\langle a^{\prime \prime \prime}, a^{\prime \prime} e^{\prime \prime}\right\rangle=\left\langle a^{\prime \prime \prime}, a^{\prime \prime}\right\rangle,
$$


showing that the weak and weak ${ }^{*}$ topologies coincide on $\mathcal{A}^{* *}$. Therefore $\mathcal{A}^{* *}$ is reflexive, and so also is $\mathcal{A}$. This proves statement (1).

For the second statement, let $\mathcal{B}:=\mathcal{A}$ with the product $\varrho=\pi^{t}$. Then $\mathcal{B}$ is a Banach algebra with a brai, and $\varrho^{*}\left(=\pi^{t *}\right)$ is Arens regular. Statement (1) implies that $\mathcal{B}$ is reflexive, and so is $\mathcal{A}$.

Corollary 2.1. Let $\mathcal{A}$ be a Banach algebra with a bai. Then the following assertions are equivalent.

(1) $Z_{\mathcal{A}^{*}}\left(\mathcal{A}^{* *}\right)=\mathcal{A}^{* *}\left(\right.$ or $\pi^{*}: \mathcal{A} \times \mathcal{A}^{*} \rightarrow \mathcal{A}^{*}$ is Arens regular $)$.

(2) $Z_{\mathcal{A}}\left(\mathcal{A}^{* * *}\right)=\mathcal{A}^{* * *}\left(\right.$ or $\pi^{t * t}: \mathcal{A}^{*} \times \mathcal{A} \rightarrow \mathcal{A}^{*}$ is Arens regular $)$.

(3) $\mathcal{A}$ is reflexive.

The following example shows that the condition that $\mathcal{A}$ has a bai is essential for the corollary above to hold.

Example 2.1. Let $X$ be a non-reflexive Banach space, $x \in X$ and $f \in$ $X^{*}$ such that $\langle f, x\rangle=1$. We define the product on $\mathcal{A}:=X$ by $a b=\langle f, b\rangle a$. Then $\mathcal{A}$ is clearly a Banach algebra with a right identity $x$ (see [6]). It is straightforward to check that the left action of $\mathcal{A}$ on $\mathcal{A}^{*}$ is given by $a a^{\prime}=\langle f, a\rangle a^{\prime}$, and so $a^{\prime \prime} a^{\prime \prime \prime}=\left\langle a^{\prime \prime}, f\right\rangle a^{\prime \prime \prime}$. The right module action of $\mathcal{A}$ on $\mathcal{A}^{*}$ is given by $a^{\prime} a=\left\langle a^{\prime}, a\right\rangle f$, and so $a^{\prime \prime \prime} a^{\prime \prime}=\left\langle a^{\prime \prime \prime}, a^{\prime \prime}\right\rangle\langle\cdot, f\rangle$. Therefore we have $Z_{\mathcal{A}^{*}}\left(\mathcal{A}^{* *}\right)=\mathcal{A}^{* *}$, but $\mathcal{A}$ is not reflexive, and $Z_{\mathcal{A}}\left(\mathcal{A}^{* * *}\right) \neq \mathcal{A}^{* * *}$ (equality would imply that the weak and weak ${ }^{*}$ topologies coincide on $\mathcal{A}^{* *}$ and force $\mathcal{A}$ to be reflexive). This means that assertion (1) is equivalent to neither (2) nor (3) even if $\mathcal{A}$ has a brai. Similarly, if we give $\mathcal{B}:=X$ the product $a b=\langle f, a\rangle b$, it is easy to see that $Z_{\mathcal{B}}\left(\mathcal{B}^{* * *}\right)=\mathcal{B}^{* * *}$, but $Z_{\mathcal{B}^{*}}\left(\mathcal{B}^{* *}\right) \neq \mathcal{B}^{* *}$. So assertion (2) is equivalent to neither (1) nor (3) even if $\mathcal{B}$ has a blai.

We know from [21] that $L^{1}(G)$ is Arens regular if and only if it is reflexive (equivalent to $G$ being finite) for every locally compact group $G$ (see [10] for more details). Since $L^{1}(G)$ has a bai, we have the following corollary.

COROLlary 2.2. Let $G$ be a locally compact group. Then the following assertions are equivalent.

(1) $Z_{L^{\infty}(G)}\left(L^{1}(G)^{* *}\right)=L^{1}(G)^{* *} \quad$ (i.e., $\pi^{t * t}: L^{\infty}(G) \times L^{1}(G) \rightarrow L^{\infty}(G)$ is Arens regular $)$.

(2) $Z_{L^{1}(G)}\left(L^{1}(G)^{* * *}\right)=L^{1}(G)^{* * *} \quad$ (i.e., $\pi^{*}: L^{1}(G) \times L^{\infty}(G) \rightarrow L^{\infty}(G)$ is Arens regular $)$.

(3) $Z_{L^{1}(G)}\left(L^{1}(G)^{* *}\right)=L^{1}(G)^{* *} \quad$ (i.e., $\pi: L^{1}(G) \times L^{1}(G) \rightarrow L^{1}(G)$ is Arens regular $)$.

(4) $G$ is finite.

It is well known that a $C^{*}$-algebra is reflexive if and only if it is of finite dimension. Since also every $C^{*}$-algebra has a bai, we have the following corollary. 
Corollary 2.3. Let $\mathcal{A}$ be a $C^{*}$-algebra. Then the following assertions are equivalent.

(1) $Z_{\mathcal{A}^{*}}\left(\mathcal{A}^{* *}\right)=\mathcal{A}^{* *}\left(\right.$ or $\pi^{*}: \mathcal{A}^{*} \times \mathcal{A} \rightarrow \mathcal{A}^{*}$ is Arens regular $)$.

(2) $Z_{\mathcal{A}}\left(\mathcal{A}^{* * *}\right)=\mathcal{A}^{* * *}\left(\right.$ or $\pi^{t * t}: \mathcal{A} \times \mathcal{A}^{*} \rightarrow \mathcal{A}^{*}$ is Arens regular $)$.

(3) $\mathcal{A}$ is of finite dimension.

We next give a theorem which will be useful later in Section 5 for finding a class of Banach algebras which are left or right strongly Arens irregular but are not strongly Arens irregular. This theorem is also a generalization of Theorem 2.1.

TheOrem 2.2. Let $X$ be a right $\mathcal{A}$-module with module action $\pi_{r}$ : $X \times \mathcal{A} \rightarrow X$. If there exists $x^{\prime} \in X^{*}$ such that $\pi_{r}^{*}\left(x^{\prime}, X\right)=\mathcal{A}^{*}$ and $\pi_{r}^{* *}\left(\mathcal{A}^{* *}, x^{\prime}\right)=X^{*}$, then $Z_{\mathcal{A}}\left(X^{* *}\right)=X$.

Proof. Let $x^{\prime \prime} \in Z_{\mathcal{A}}\left(X^{* *}\right)$. We show that $x^{\prime \prime}$ is weak ${ }^{*}$ continuous from $X^{*}$ to $\mathbb{C}$, so let $y_{\alpha}^{\prime} \stackrel{\text { weak }^{*}}{\longrightarrow} y^{\prime}$ in $X^{*}$. By the assumed factorization, there exists a net $\left(b_{\alpha}^{\prime \prime}\right)$ in $\mathcal{A}^{* *}$ and $b^{\prime \prime} \in \mathcal{A}^{* *}$ such that $y_{\alpha}^{\prime}=\pi_{r}^{* *}\left(b_{\alpha}^{\prime \prime}, x^{\prime}\right)$ and $y^{\prime}=\pi_{r}^{* *}\left(b^{\prime \prime}, x^{\prime}\right)$. We show first that $b_{\alpha}^{\prime \prime} \stackrel{\text { weak }^{*}}{\longrightarrow} b^{\prime \prime}$ in $\mathcal{A}^{* *}$. So let $a^{\prime} \in \mathcal{A}^{*}$ and pick $x \in X$ such that $a^{\prime}=\pi_{r}^{*}\left(x^{\prime}, x\right)$. Then

$$
\begin{aligned}
\lim _{\alpha}\left\langle b_{\alpha}^{\prime \prime}, a^{\prime}\right\rangle & =\lim _{\alpha}\left\langle b_{\alpha}^{\prime \prime}, \pi_{r}^{*}\left(x^{\prime}, x\right)\right\rangle=\lim _{\alpha}\left\langle\pi_{r}^{* *}\left(b_{\alpha}^{\prime \prime}, x^{\prime}\right), x\right\rangle=\lim _{\alpha}\left\langle y_{\alpha}^{\prime}, x\right\rangle \\
& =\left\langle y^{\prime}, x\right\rangle=\left\langle\pi_{r}^{* *}\left(b^{\prime \prime}, x^{\prime}\right), x\right\rangle=\left\langle b^{\prime \prime}, \pi_{r}^{*}\left(x^{\prime}, x\right)\right\rangle=\left\langle b^{\prime \prime}, a^{\prime}\right\rangle .
\end{aligned}
$$

Thus $b_{\alpha}^{\prime \prime} \stackrel{\text { weak }^{*}}{\longrightarrow} b^{\prime \prime}$ in $\mathcal{A}^{* *}$. Now since $x^{\prime \prime} \in Z_{\mathcal{A}}\left(X^{* *}\right)$, we have

$$
\begin{aligned}
\lim _{\alpha}\left\langle x^{\prime \prime}, y_{\alpha}^{\prime}\right\rangle & =\lim _{\alpha}\left\langle x^{\prime \prime}, \pi_{r}^{* *}\left(b_{\alpha}^{\prime \prime}, x^{\prime}\right)\right\rangle=\lim _{\alpha}\left\langle\pi_{r}^{* * *}\left(x^{\prime \prime}, b_{\alpha}^{\prime \prime}\right), x^{\prime}\right\rangle \\
& =\left\langle\pi_{r}^{* * *}\left(x^{\prime \prime}, b^{\prime \prime}\right), x^{\prime}\right\rangle=\left\langle x^{\prime \prime}, \pi_{r}^{* *}\left(b^{\prime \prime}, x^{\prime}\right)\right\rangle=\left\langle x^{\prime \prime}, y^{\prime}\right\rangle .
\end{aligned}
$$

Since $x^{\prime \prime}: X^{*} \rightarrow \mathbb{C}$ is weak ${ }^{*}$ continuous, it must be in $X$. The proof is complete.

Corollary 2.4. Let $\mathcal{A}$ be a Banach algebra with a brai. Then $Z_{\mathcal{A}}\left(\mathcal{A}^{* * *}\right)$ $=\mathcal{A}^{*}$.

Proof. Let $\pi: \mathcal{A} \times \mathcal{A} \rightarrow \mathcal{A}$ be the product of $\mathcal{A}$ and let $e^{\prime \prime}$ be a cluster point of a brai of $\mathcal{A}$ in $\mathcal{A}^{* *}$. Then $\pi_{r}^{*}\left(e^{\prime \prime}, \mathcal{A}^{*}\right)=\mathcal{A}^{*}$ and $\pi_{r}^{* *}\left(\mathcal{A}^{* *}, e^{\prime \prime}\right)=\mathcal{A}^{* *}$. Applying the theorem above, we obtain $Z_{\mathcal{A}}\left(\mathcal{A}^{* * *}\right)=\mathcal{A}^{*}$.

3. Arens regularity of the left module action of $\mathcal{A}$ on $\mathcal{A}^{(n)}$. In this section, we relate the Arens regularity and strong Arens irregularity of the left module actions of $\mathcal{A}$ on the $n$th dual $\mathcal{A}^{(n)}$ of $\mathcal{A}$ to the Arens regularity and strong Arens irregularity of $\mathcal{A}$. We prove, in particular, that not only is $L^{1}(G)$ strongly Arens irregular but also $Z_{L^{1}(G)^{(n)}}\left(L^{1}(G)^{* *}\right)=L^{1}(G)$ for every $n \in \mathbb{N}$. 
Let $X, Y$ and $Z$ be normed spaces and let $f: X \times Y \rightarrow Z$ be a continuous bilinear map. Then we say that $f$ factors if $f$ is onto $Z$.

Theorem 3.1. Let $\mathcal{A}$ be a Banach algebra and let $X$ be a left Banach $\mathcal{A}$-module with module action $\pi_{l}: \mathcal{A} \times X \rightarrow X$. If $\pi_{l}^{* *}: X^{* *} \times X^{*} \rightarrow \mathcal{A}^{*}$ factors, then $Z_{X}\left(\mathcal{A}^{* *}\right) \subseteq Z_{\mathcal{A}}\left(\mathcal{A}^{* *}\right)$.

To prove this theorem, we need the following lemma.

Lemma 3.1. Let $\pi: \mathcal{A} \times \mathcal{A} \rightarrow \mathcal{A}$ be the product of $\mathcal{A}$, and let $X$ be a left Banach $\mathcal{A}$-module with module action $\pi_{l}: \mathcal{A} \times X \rightarrow X$. Then

(1) $\pi^{*}\left(\pi_{l}^{* *}\left(x^{\prime \prime}, x^{\prime}\right), a\right)=\pi_{l}^{* *}\left(x^{\prime \prime}, \pi_{l}^{*}\left(x^{\prime}, a\right)\right)\left(a \in \mathcal{A}, x^{\prime \prime} \in X^{* *}, x^{\prime} \in X^{*}\right)$,

(2) $\pi_{l}^{* *}\left(\pi_{l}^{* * *}\left(b^{\prime \prime}, x^{\prime \prime}\right), x^{\prime}\right)=\pi^{* *}\left(b^{\prime \prime}, \pi_{l}^{* *}\left(x^{\prime \prime}, x^{\prime}\right)\right)\left(b^{\prime \prime} \in \mathcal{A}^{* *}, x^{\prime \prime} \in X^{* *}\right.$, $\left.x^{\prime} \in X^{*}\right)$.

Proof. Let $a, b \in \mathcal{A}, x \in X, x^{\prime} \in X^{*}, x^{\prime \prime} \in X^{* *}$, and note first that

$$
\begin{aligned}
\left\langle\pi_{l}^{*}\left(x^{\prime}, \pi(a, b), x\right\rangle\right. & =\left\langle x^{\prime}, \pi_{l}(\pi(a, b), x)\right\rangle=\left\langle x^{\prime}, \pi_{l}\left(a, \pi_{l}(b, x)\right)\right\rangle \\
& =\left\langle\pi_{l}^{*}\left(x^{\prime}, a\right), \pi_{l}(b, x)\right\rangle=\left\langle\pi_{l}^{*}\left(\pi_{l}^{*}\left(x^{\prime}, a\right), b\right), x\right\rangle
\end{aligned}
$$

for every $x \in X$, showing that $\pi_{l}^{*}\left(x^{\prime}, \pi(a, b)=\pi_{l}^{*}\left(\pi_{l}^{*}\left(x^{\prime}, a\right), b\right)\right.$. It follows that

$$
\begin{aligned}
\left\langle\pi^{*}\left(\pi_{l}^{* *}\left(x^{\prime \prime}, x^{\prime}\right), a\right), b\right\rangle & =\left\langle\pi_{l}^{* *}\left(x^{\prime \prime}, x^{\prime}\right), \pi(a, b)\right\rangle=\left\langle x^{\prime \prime}, \pi_{l}^{*}\left(x^{\prime}, \pi(a, b)\right\rangle\right. \\
& =\left\langle x^{\prime \prime}, \pi_{l}^{*}\left(\pi_{l}^{*}\left(x^{\prime}, a\right), b\right)\right\rangle=\left\langle\pi_{l}^{* *}\left(x^{\prime \prime}, \pi_{l}^{*}\left(x^{\prime}, a\right)\right), b\right\rangle,
\end{aligned}
$$

which proves (1).

To prove (2), we apply (1):

$$
\begin{aligned}
\left\langle\pi_{l}^{* *}\left(\pi_{l}^{* * *}\left(b^{\prime \prime}, x^{\prime \prime}\right), x^{\prime}\right), a\right\rangle & =\left\langle\pi_{l}^{* * *}\left(b^{\prime \prime}, x^{\prime \prime}\right), \pi_{l}^{*}\left(x^{\prime}, a\right)\right\rangle=\left\langle b^{\prime \prime}, \pi_{l}^{* *}\left(x^{\prime \prime}, \pi_{l}^{*}\left(x^{\prime}, a\right)\right\rangle\right. \\
& =\left\langle b^{\prime \prime}, \pi^{*}\left(\pi_{l}^{* *}\left(x^{\prime \prime}, x^{\prime}\right), a\right)\right\rangle=\left\langle\pi^{* *}\left(b^{\prime \prime}, \pi_{l}^{* *}\left(x^{\prime \prime}, x^{\prime}\right)\right), a\right\rangle,
\end{aligned}
$$

as required.

Proof of Theorem 3.1. Let $\pi: \mathcal{A} \times \mathcal{A} \rightarrow \mathcal{A}$ be the product of $\mathcal{A}, a^{\prime \prime} \in$ $Z_{X}\left(\mathcal{A}^{* *}\right)$, and let $b_{\alpha}^{\prime \prime} \stackrel{\text { weak }^{*}}{\longrightarrow} b^{\prime \prime}$ in $\mathcal{A}^{* *}$. We must show that $\pi^{* * *}\left(a^{\prime \prime}, b_{\alpha}^{\prime \prime}\right) \stackrel{\text { weak }^{*}}{\longrightarrow}$ $\pi^{* * *}\left(a^{\prime \prime}, b^{\prime \prime}\right)$ in $\mathcal{A}^{* *}$. So let $a^{\prime} \in \mathcal{A}^{*}$. Since $\pi_{l}^{* *}$ factors, there exist $x^{\prime \prime} \in X^{* *}$ and $x^{\prime} \in X^{*}$ such that $a^{\prime}=\pi_{l}^{* *}\left(x^{\prime \prime}, x^{\prime}\right)$. Since we know that $\pi_{l}^{* * *}\left(b_{\alpha}^{\prime \prime}, x^{\prime \prime}\right) \stackrel{\text { weak }^{*}}{\longrightarrow}$ $\pi_{l}^{* * *}\left(b^{\prime \prime}, x^{\prime \prime}\right)$ in $X^{* *}$, we see that

$$
\pi_{l}^{* * *}\left(a^{\prime \prime}, \pi_{l}^{* * *}\left(b_{\alpha}^{\prime \prime}, x^{\prime \prime}\right)\right) \stackrel{\text { weak }^{*}}{\longrightarrow} \pi_{l}^{* * *}\left(a^{\prime \prime}, \pi_{l}^{* * *}\left(b^{\prime \prime}, x^{\prime \prime}\right)\right)
$$

in $X^{* *}$ (since $\left.a^{\prime \prime} \in Z_{X}\left(\mathcal{A}^{* *}\right)\right)$. Therefore by Lemma 3.1(2), we have

$$
\begin{aligned}
\lim _{\alpha}\left\langle a^{\prime \prime} b_{\alpha}^{\prime \prime}, a^{\prime}\right\rangle & =\lim _{\alpha}\left\langle a^{\prime \prime}, \pi^{* *}\left(b_{\alpha}^{\prime \prime}, a^{\prime}\right)\right\rangle=\lim _{\alpha}\left\langle a^{\prime \prime}, \pi^{* *}\left(b_{\alpha}^{\prime \prime}, \pi_{l}^{* *}\left(x^{\prime \prime}, x^{\prime}\right)\right)\right\rangle \\
& =\lim _{\alpha}\left\langle a^{\prime \prime}, \pi_{l}^{* *}\left(\pi_{l}^{* * *}\left(b_{\alpha}^{\prime \prime}, x^{\prime \prime}\right), x^{\prime}\right)\right\rangle=\lim _{\alpha}\left\langle\pi_{l}^{* * *}\left(a^{\prime \prime}, \pi_{l}^{* * *}\left(b_{\alpha}^{\prime \prime}, x^{\prime \prime}\right)\right), x^{\prime}\right\rangle \\
& =\left\langle\pi_{l}^{* * *}\left(a^{\prime \prime}, \pi_{l}^{* * *}\left(b^{\prime \prime}, x^{\prime \prime}\right)\right), x^{\prime}\right\rangle=\left\langle a^{\prime \prime}, \pi_{l}^{* *}\left(\pi_{l}^{* * *}\left(b^{\prime \prime}, x^{\prime \prime}\right), x^{\prime}\right)\right\rangle \\
& =\left\langle a^{\prime \prime}, \pi^{* *}\left(b^{\prime \prime}, \pi_{l}^{* *}\left(x^{\prime \prime}, x^{\prime}\right)\right\rangle=\left\langle a^{\prime \prime}, \pi^{* *}\left(b^{\prime \prime}, a^{\prime}\right)\right\rangle=\left\langle a^{\prime \prime} b^{\prime \prime}, a^{\prime}\right\rangle .\right.
\end{aligned}
$$

Therefore $\pi^{* * *}\left(a^{\prime \prime}, b_{\alpha}^{\prime \prime}\right) \stackrel{\text { weak }^{*}}{\longrightarrow} \pi^{* * *}\left(a^{\prime \prime}, b^{\prime \prime}\right)$ in $\mathcal{A}^{* *}$, as required. 
Lemma 3.2. Let $\mathcal{A}$ be a Banach algebra, let $\pi_{l_{n}}: \mathcal{A} \times \mathcal{A}^{(n)} \rightarrow \mathcal{A}^{(n)}$ be the left module action of $\mathcal{A}$ on $\mathcal{A}^{(n)}$, and consider $\pi_{l_{n}}^{* *}: \mathcal{A}^{(n+2)} \times \mathcal{A}^{(n+1)} \rightarrow \mathcal{A}^{*}$.

(1) If $\mathcal{A}$ has a blai, then $\pi_{l_{n}}^{* *}$ factors for every positive odd integer $n$.

(2) If $\mathcal{A}$ has a brai, then $\pi_{l_{n}}^{* *}$ factors for every positive even integer $n$.

Proof. (1) Let $\left(e_{\alpha}\right)$ be a blai in $\mathcal{A}$ with a cluster point $e^{\prime \prime} \in \mathcal{A}^{* *}$. We argue by induction and prove that $\pi_{l_{2 k-1}}^{* *}\left(a^{\prime}, e^{\prime \prime}\right)=a^{\prime}$ for every $k \in \mathbb{N}$ and $a^{\prime} \in \mathcal{A}^{*}$. As usual we start with $k=1$, and let $a \in \mathcal{A}$ be arbitrary. Then

$$
\begin{aligned}
\left\langle\pi_{l_{1}}^{* *}\left(a^{\prime}, e^{\prime \prime}\right), a\right\rangle & =\left\langle a^{\prime}, \pi_{l_{1}}^{*}\left(e^{\prime \prime}, a\right)\right\rangle=\left\langle\pi_{l_{1}}^{*}\left(e^{\prime \prime}, a\right), a^{\prime}\right\rangle \\
& =\left\langle e^{\prime \prime}, \pi_{l_{1}}\left(a, a^{\prime}\right)\right\rangle=\lim _{\alpha}\left\langle e_{\alpha}, \pi_{l_{1}}\left(a, a^{\prime}\right)\right\rangle \\
& =\lim _{\alpha}\left\langle\pi_{l_{1}}\left(a, a^{\prime}\right), e_{\alpha}\right\rangle=\lim _{\alpha}\left\langle a^{\prime}, e_{\alpha} a\right\rangle=\left\langle a^{\prime}, a\right\rangle,
\end{aligned}
$$

hence $\pi_{l_{1}}^{* *}\left(a^{\prime}, e^{\prime \prime}\right)=a^{\prime}$, and so $\pi_{l_{1}}^{* *}$ factors.

Now we show, that for any $a^{\prime} \in \mathcal{A}^{*}$ and $a \in \mathcal{A}$, we have

$$
\left\langle\pi_{l_{2 k+1}}^{* *}\left(a^{\prime}, e^{\prime \prime}\right), a\right\rangle=\left\langle\pi_{l_{2 k-1}}^{* *}\left(a^{\prime}, e^{\prime \prime}\right), a\right\rangle \quad(k \geq 1),
$$

which will clearly yield our claim. Using $\pi_{l_{n}}=\pi_{r_{n-1}}^{t * t}$ and $\pi_{r_{n}}=\pi_{l_{n-1}}^{*}$ for every $n \geq 1$, we obtain

$$
\begin{aligned}
\left\langle\pi_{l_{2 k+1}}^{* *}\left(a^{\prime}, e^{\prime \prime}\right), a\right\rangle & =\left\langle\pi_{l_{2 k+1}}^{*}\left(e^{\prime \prime}, a\right), a^{\prime}\right\rangle=\left\langle e^{\prime \prime}, \pi_{l_{2 k+1}}\left(a, a^{\prime}\right)\right\rangle=\left\langle a^{\prime}, \pi_{r_{2 k}}\left(e^{\prime \prime}, a\right)\right\rangle \\
& =\left\langle\pi_{r_{2 k}}\left(e^{\prime \prime}, a\right), a^{\prime}\right\rangle=\left\langle e^{\prime \prime}, \pi_{l_{2 k-1}}\left(a, a^{\prime}\right)\right\rangle=\left\langle\pi_{l_{2 k-1}}^{*}\left(e^{\prime \prime}, a\right), a^{\prime}\right\rangle \\
& =\left\langle a^{\prime}, \pi_{l_{2 k-1}}^{*}\left(e^{\prime \prime}, a\right)\right\rangle=\left\langle\pi_{l_{2 k-1}}^{* *}\left(a^{\prime}, e^{\prime \prime}\right), a\right\rangle,
\end{aligned}
$$

as required. Therefore, for every odd integer $n \geq 1, \pi_{l_{n}}^{* *}$ factors.

(2) Suppose now that $e^{\prime \prime}$ is a cluster point in $\mathcal{A}^{* *}$ of a brai $\left(e_{\alpha}\right)$ of $\mathcal{A}$. Then, for $n=2$, we have

$$
\begin{aligned}
\left\langle\pi_{l_{2}}^{* *}\left(e^{\prime \prime}, a^{\prime}\right), a\right\rangle & =\left\langle e^{\prime \prime}, \pi_{l_{2}}^{*}\left(a^{\prime}, a\right)\right\rangle=\left\langle\pi_{l_{2}}^{*}\left(a^{\prime}, a\right), e^{\prime \prime}\right\rangle=\left\langle a^{\prime}, \pi_{l_{2}}\left(a, e^{\prime \prime}\right)\right\rangle \\
& =\left\langle\pi_{l_{2}}\left(a, e^{\prime \prime}\right), a^{\prime}\right\rangle=\left\langle e^{\prime \prime}, \pi_{r_{1}}\left(a^{\prime}, a\right)\right\rangle=\lim _{\alpha}\left\langle e_{\alpha}, \pi_{r_{1}}\left(a^{\prime}, a\right)\right\rangle \\
& =\lim _{\alpha}\left\langle\pi_{r_{1}}\left(a^{\prime}, a\right), e_{\alpha}\right\rangle=\lim _{\alpha}\left\langle a^{\prime}, a e_{\alpha}\right\rangle=\left\langle a^{\prime}, a\right\rangle,
\end{aligned}
$$

and so $\pi_{l_{2}}^{* *}$ factors. As before, we next show that

$$
\left\langle\pi_{l_{2 k}}^{* *}\left(e^{\prime \prime}, a^{\prime}\right), a\right\rangle=\left\langle\pi_{l_{2 k-2}}^{* *}\left(e^{\prime \prime}, a^{\prime}\right), a\right\rangle \quad(k \geq 2) .
$$

Using $\pi_{l_{n}}=\pi_{r_{n-1}}^{t * t}$ and $\pi_{r_{n}}=\pi_{l_{n-1}}^{*}$ for every $n \geq 1$, we obtain

$$
\begin{aligned}
\left\langle\pi_{l_{2 k}}^{* *}\left(e^{\prime \prime}, a^{\prime}\right), a\right\rangle & \left.=\left\langle e^{\prime \prime}, \pi_{l_{2 k}}^{*}\left(a^{\prime}, a\right)\right\rangle=\left\langle\pi_{l_{2 k}}^{*}\left(a^{\prime}, a\right), e^{\prime \prime}\right)\right\rangle=\left\langle a^{\prime}, \pi_{l_{2 k}}\left(a, e^{\prime \prime}\right)\right\rangle \\
& =\left\langle\pi_{l_{2 k}}\left(a, e^{\prime \prime}\right), a^{\prime}\right\rangle=\left\langle e^{\prime \prime}, \pi_{r_{2 k-1}}\left(a^{\prime}, a\right)\right\rangle=\left\langle\pi_{r_{2 k-1}}\left(a^{\prime}, a\right), e^{\prime \prime}\right\rangle \\
& =\left\langle a^{\prime}, \pi_{l_{2 k-2}}\left(a, e^{\prime \prime}\right)\right\rangle=\left\langle\pi_{l_{2 k-2}}^{*}\left(a^{\prime}, a\right), e^{\prime \prime}\right\rangle=\left\langle e^{\prime \prime}, \pi_{l_{2 k-2}}^{*}\left(a^{\prime}, a\right)\right\rangle \\
& =\left\langle\pi_{l_{2 k-2}}^{* *}\left(e^{\prime \prime}, a^{\prime}\right), a\right\rangle .
\end{aligned}
$$

Therefore, $\pi_{l_{2 k}}^{* *}\left(e^{\prime \prime}, a^{\prime}\right)=\pi_{l_{2 k-2}}^{* *}\left(e^{\prime \prime}, a^{\prime}\right)$, implying by induction that $\pi_{l_{n}}^{* *}$ factors for every even integer $n \geq 2$. 
Theorem 3.2. Let $\mathcal{A}$ be a Banach algebra and $k \geq 1$.

(1) If $\mathcal{A}$ has a blai, then $Z_{\mathcal{A}^{(2 k-1)}}\left(\mathcal{A}^{* *}\right) \subseteq Z_{\mathcal{A}}\left(\mathcal{A}^{* *}\right)$.

(2) If $\mathcal{A}$ has a brai, then $Z_{\mathcal{A}^{(2 k)}}\left(\mathcal{A}^{* *}\right) \subseteq Z_{\mathcal{A}}\left(\mathcal{A}^{* *}\right)$.

Proof. Apply Theorem 3.1 and Lemma 3.2.

Corollary 3.1. If $\mathcal{A}$ has a brai (blai) and the left module action of $\mathcal{A}$ on $\mathcal{A}^{(n)}$ is Arens regular for some positive even (odd) integer n, then $\mathcal{A}$ is Arens regular.

Corollary 3.2. If $\mathcal{A}$ has a bai and is left strongly Arens irregular, then for every $n \geq 1$, the left module action of $\mathcal{A}$ on $\mathcal{A}^{(n)}$ is strongly Arens irregular, i.e., $Z_{\mathcal{A}^{(n)}}\left(\mathcal{A}^{* *}\right)=\mathcal{A}$.

We know that for every locally compact group $G$, the group algebra $L^{1}(G)$ is left strongly Arens irregular (see [13], [9], [15]). So we have the following result.

Corollary 3.3. Let $G$ be a locally compact group and $n \geq 1$. Then $Z_{L^{1}(G)^{(n)}}\left(L^{1}(G)^{* *}\right)=L^{1}(G)$.

Remark. The inclusions in Theorems 3.1 and 3.2 may be strict, as can be seen from Corollary 2.3 for infinite-dimensional $C^{*}$-algebras.

4. Arens regularity and some factorizations. Let $\mathcal{A}$ be a Banach algebra and $X$ be a Banach $\mathcal{A}$-bimodule. Then $X$ factors $\mathcal{A}$ on the left (right) if $X=X \mathcal{A}(X=\mathcal{A} X)$. In this section, we see in particular how Arens regularity is implied by the factorization of $\mathcal{A}^{*}$ or $\mathcal{A}^{* *}$ when $\mathcal{A}$ is a left or right ideal in $\mathcal{A}^{* *}$. When $\mathcal{A}$ has a bai with a weak* cluster point $e^{\prime \prime}$ in $\mathcal{A}^{* *}$, Lau and Ülger related in [14] the factorization of $\mathcal{A}^{*}$ to $e^{\prime \prime}$. Here we obtain some results relating the factorization of $\mathcal{A}^{* *}$ to $e^{\prime \prime}$.

Theorem 4.1. Let $\mathcal{A}$ be a Banach algebra, $X$ be a Banach $\mathcal{A}$-bimodule and regard $X^{*}$ as a Banach $\mathcal{A}$-bimodule. If $\mathcal{A}$ is a left ideal in $\mathcal{A}^{* *}$ and $X^{*}$ factors $\mathcal{A}$ on the right, then $Z_{\mathcal{A}}\left(X^{* *}\right)=X^{* *}$.

Proof. Let $x^{\prime \prime} \in X^{* *}$ and suppose that $a_{\alpha}^{\prime \prime} \stackrel{\text { weak }^{*}}{\longrightarrow} a^{\prime \prime}$ in $\mathcal{A}^{* *}$. We show that $x^{\prime \prime} a_{\alpha}^{\prime \prime} \stackrel{\text { weak }}{\longrightarrow} x^{\prime \prime} a^{\prime \prime}$ in $X^{* *}$. Let $x^{\prime} \in X^{*}$. Then there exist $a \in \mathcal{A}$ and $y^{\prime} \in X^{*}$ such that $x^{\prime}=a y^{\prime}$. Since $\mathcal{A}$ is a left ideal in $\mathcal{A}^{* *}$, we have $a_{\alpha}^{\prime \prime} a \stackrel{\text { weak }^{*}}{\longrightarrow} a^{\prime \prime} a$ in $\mathcal{A}^{* *}$ if and only if $a_{\alpha}^{\prime \prime} a \stackrel{\text { weak }}{\longrightarrow} a^{\prime \prime} a$ in $\mathcal{A}$. Since $a \mapsto a y^{\prime}: \mathcal{A} \rightarrow X^{*}$ is weak-weak continuous (see for example [8, Theorem V.3.15]), it follows that $a_{\alpha}^{\prime \prime} a y^{\prime} \stackrel{\text { weak }}{\longrightarrow} a^{\prime \prime} a y^{\prime}$ in $X^{*}$. Thus

$$
\lim _{\alpha}\left\langle x^{\prime \prime} a_{\alpha}^{\prime \prime}, x^{\prime}\right\rangle=\lim _{\alpha}\left\langle x^{\prime \prime} a_{\alpha}^{\prime \prime}, a y^{\prime}\right\rangle=\lim _{\alpha}\left\langle x^{\prime \prime}, a_{\alpha}^{\prime \prime} a y^{\prime}\right\rangle=\left\langle x^{\prime \prime}, a^{\prime \prime} a y^{\prime}\right\rangle=\left\langle x^{\prime \prime} a^{\prime \prime}, x^{\prime}\right\rangle,
$$

showing that $x^{\prime \prime} \in Z_{\mathcal{A}}\left(X^{* *}\right)$. 
Corollary 4.1. Let $\mathcal{A}$ be a left ideal in $\mathcal{A}^{* *}$.

(1) If $\mathcal{A}^{*}$ factors $\mathcal{A}$ on the right, then $\mathcal{A}$ is Arens regular.

(2) If $\mathcal{A}^{* *}$ factors $\mathcal{A}$ on the right, then $Z_{\mathcal{A}}\left(\mathcal{A}^{* * *}\right)=\mathcal{A}^{* * *}$.

Theorem 4.2. Let $\mathcal{A}$ be a right ideal in $\mathcal{A}^{* *}$. In each of the following situations, $\mathcal{A}$ is Arens regular.

(1) $\mathcal{A}^{*}$ factors $\mathcal{A}$ on the left.

(2) $\mathcal{A}^{* *}$ factors $\mathcal{A}$ on the left.

Proof. Let $a^{\prime \prime} \in \mathcal{A}^{* *}$ and let $b_{\alpha}^{\prime \prime} \stackrel{\text { weak }}{\longrightarrow} b^{\prime \prime}$ in $\mathcal{A}^{* *}$. In each situation, we must show that $a^{\prime \prime} b_{\alpha}^{\prime \prime} \stackrel{\text { weak }^{*}}{\longrightarrow} a^{\prime \prime} b^{\prime \prime}$ in $\mathcal{A}^{* *}$. For $(1)$, let $a^{\prime} \in \mathcal{A}^{*}$ and pick $b^{\prime} \in \mathcal{A}^{*}$ and $b \in \mathcal{A}$ such that $a^{\prime}=b^{\prime} b$. Then, since $\mathcal{A}$ is a right ideal in $\mathcal{A}^{* *}$,

$$
\begin{aligned}
\lim _{\alpha}\left\langle a^{\prime \prime} b_{\alpha}^{\prime \prime}, a^{\prime}\right\rangle & =\lim _{\alpha}\left\langle a^{\prime \prime} b_{\alpha}^{\prime \prime}, b^{\prime} b\right\rangle=\lim _{\alpha}\left\langle b a^{\prime \prime} b_{\alpha}^{\prime \prime}, b^{\prime}\right\rangle \\
& =\left\langle b a^{\prime \prime} b^{\prime \prime}, b^{\prime}\right\rangle=\left\langle a^{\prime \prime} b^{\prime \prime}, b^{\prime} b\right\rangle=\left\langle a^{\prime \prime} b^{\prime \prime}, a^{\prime}\right\rangle,
\end{aligned}
$$

showing that $a^{\prime \prime} \in Z\left(\mathcal{A}^{* *}\right)$. Thus $\mathcal{A}$ is Arens regular.

For the second situation, pick $c^{\prime \prime} \in \mathcal{A}^{* *}$ and $a \in \mathcal{A}$ such that $a^{\prime \prime}=c^{\prime \prime} a$. Since $\mathcal{A}$ is a right ideal in $\mathcal{A}^{* *}$, we see that $a b_{\alpha}^{\prime \prime} \stackrel{\text { weak }}{\longrightarrow} a b^{\prime \prime}$ in $\mathcal{A}$, and so again by [8, Theorem V.3.15], $a b_{\alpha}^{\prime \prime} a^{\prime} \stackrel{\text { weak }}{\longrightarrow} a b^{\prime \prime} a^{\prime}$ in $\mathcal{A}^{*}$ for every $a^{\prime} \in \mathcal{A}^{*}$. Thus

$$
\begin{aligned}
\lim _{\alpha}\left\langle a^{\prime \prime} b_{\alpha}^{\prime \prime}, a^{\prime}\right\rangle & =\lim _{\alpha}\left\langle a^{\prime \prime}, b_{\alpha}^{\prime \prime} a^{\prime}\right\rangle=\lim _{\alpha}\left\langle c^{\prime \prime}, a b_{\alpha}^{\prime \prime} a^{\prime}\right\rangle \\
& =\left\langle c^{\prime \prime}, a b^{\prime \prime} a^{\prime}\right\rangle=\left\langle\left(c^{\prime \prime} a\right) b^{\prime \prime}, a^{\prime}\right\rangle=\left\langle a^{\prime \prime} b^{\prime \prime}, a^{\prime}\right\rangle,
\end{aligned}
$$

showing again that $a^{\prime \prime} \in Z\left(\mathcal{A}^{* *}\right)$. Therefore $\mathcal{A}$ is Arens regular.

In $\left[6\right.$, Section 5], it was proved that for a Banach algebra $\mathcal{A}, \mathcal{A}^{*}$ is an $\mathcal{A}^{* *}$-submodule of $\mathcal{A}^{* * *}$ if and only if $\mathcal{A}$ is Arens regular. Below we show that in fact $\mathcal{A}^{*}$ is always a $Z_{\mathcal{A}}\left(\mathcal{A}^{* *}\right)$-submodule of $\mathcal{A}^{* * *}$.

TheOREM 4.3. Let $\mathcal{A}$ be a Banach algebra. Then the following assertions hold.

(1) $\mathcal{A}^{*}$ is always a $Z_{\mathcal{A}}\left(\mathcal{A}^{* *}\right)$-submodule of $\mathcal{A}^{* * *}$.

(2) If $\mathcal{A}^{*}$ factors $Z_{\mathcal{A}}\left(\mathcal{A}^{* *}\right)$ on the left and $Z_{\mathcal{A}}\left(\mathcal{A}^{* *}\right)$ is a right ideal of $\mathcal{A}^{* *}$, then $\mathcal{A}$ is Arens regular.

Proof. For (1), let $a^{\prime} \in \mathcal{A}^{*}$ and $a^{\prime \prime} \in Z_{\mathcal{A}}\left(\mathcal{A}^{* *}\right)$. We verify that $a^{\prime} a^{\prime \prime}$ as an element of $\mathcal{A}^{* * *}$ is weak* continuous from $\mathcal{A}^{* *}$ into $\mathbb{C}$, and so it must be in $\mathcal{A}^{*}$. Let $b_{\alpha}^{\prime \prime} \stackrel{\text { weak }^{*}}{\longrightarrow} b^{\prime \prime}$ in $\mathcal{A}^{* *}$. Then

$$
\lim _{\alpha}\left\langle a^{\prime} a^{\prime \prime}, b_{\alpha}^{\prime \prime}\right\rangle=\lim _{\alpha}\left\langle a^{\prime}, a^{\prime \prime} b_{\alpha}^{\prime \prime}\right\rangle=\left\langle a^{\prime}, a^{\prime \prime} b^{\prime \prime}\right\rangle=\left\langle a^{\prime} a^{\prime \prime}, b^{\prime \prime}\right\rangle,
$$

as required.

With statement (1) in mind, the proof of statement (2) is the same as that of statement (1) in Theorem 4.2 with $Z_{\mathcal{A}}\left(\mathcal{A}^{* *}\right)$ replacing $\mathcal{A}$. 
It is known that if a Banach algebra $\mathcal{A}$ with a bai is a right ideal in $\mathcal{A}^{* *}$ then $\operatorname{WAP}(\mathcal{A})=\mathcal{A}^{*} \mathcal{A}$ (see [20]). But the converse is not true since $\mathrm{WAP}(\mathcal{A})=\mathcal{A}^{*} \mathcal{A}=\mathcal{A}^{*}$ whenever $\mathcal{A}$ is Arens regular. This has been adjusted in $\left[14\right.$, Theorem 3.6] by showing that $\operatorname{WAP}(\mathcal{A})=\mathcal{A}^{*} \mathcal{A}$ if and only if $\mathcal{A} \mathcal{A}^{* *} \subseteq$ $Z_{\mathcal{A}}\left(\mathcal{A}^{* *}\right)$. Next we further improve this theorem.

Theorem 4.4. Let the Banach algebra $\mathcal{A}$ have a bai. If $Z_{\mathcal{A}}\left(\mathcal{A}^{* *}\right)$ is a right ideal of $\mathcal{A}^{* *}$, then $\operatorname{WAP}(\mathcal{A})=\mathcal{A}^{*} \mathcal{A}$. If , in addition, $Z_{\mathcal{A}}\left(\mathcal{A}^{* *}\right)$ factors $\mathcal{A}$ on the left, then these statements are equivalent.

Proof. Since $Z_{\mathcal{A}}\left(\mathcal{A}^{* *}\right)$ is a right ideal in $\mathcal{A}^{* *}$, we see that

$$
\mathcal{A} \mathcal{A}^{* *} \subseteq Z_{\mathcal{A}}\left(\mathcal{A}^{* *}\right) \mathcal{A}^{* *} \subseteq Z_{\mathcal{A}}\left(\mathcal{A}^{* *}\right)
$$

Applying [14, Theorem 3.6], we obtain $\operatorname{WAP}(\mathcal{A})=\mathcal{A}^{*} \mathcal{A}$.

Suppose now that $Z_{\mathcal{A}}\left(\mathcal{A}^{* *}\right)$ factors $\mathcal{A}$ on the left and $\operatorname{WAP}(\mathcal{A})=\mathcal{A}^{*} \mathcal{A}$. Then, by [14, Theorem 3.6], $\mathcal{A A}^{* *} \subseteq Z_{\mathcal{A}}\left(\mathcal{A}^{* *}\right)$. Let now $a^{\prime \prime} \in \mathcal{A}^{* *}$ and $b^{\prime \prime} \in Z_{\mathcal{A}}\left(\mathcal{A}^{* *}\right)$, and pick $c^{\prime \prime} \in Z_{\mathcal{A}}\left(\mathcal{A}^{* *}\right)$ and $a \in \mathcal{A}$ such that $b^{\prime \prime}=c^{\prime \prime} a$. Then we have $b^{\prime \prime} a^{\prime \prime}=c^{\prime \prime}\left(a a^{\prime \prime}\right) \in Z_{\mathcal{A}}\left(\mathcal{A}^{* *}\right)$.

Theorem 4.5. Let the Banach algebra $\mathcal{A}$ have a brai $\left(e_{\alpha}\right)$ and let $X$ be a right (left) Banach $\mathcal{A}$-module. Then $X$ factors $\mathcal{A}$ on the left (on the right) if and only if $x e_{\alpha} \stackrel{\text { weak }}{\longrightarrow} x\left(e_{\alpha} x \stackrel{\text { weak }}{\longrightarrow} x\right)$ in $X$ for every $x \in X$.

Proof. Suppose that $X$ factors $\mathcal{A}$ on the left, let $x$ be any element in $X$ and write it as $x=y a$ for some $y \in X$ and $a \in \mathcal{A}$. Note first that since $a e_{\alpha} \stackrel{\|\cdot\|}{\longrightarrow} a$ in $\mathcal{A}$, we have $a e_{\alpha} \stackrel{\text { weak }}{\longrightarrow} a$ in $\mathcal{A}$. Accordingly, for any $x^{\prime} \in X^{*}$,

$$
\lim _{\alpha}\left\langle x e_{\alpha}, x^{\prime}\right\rangle=\lim _{\alpha}\left\langle y a e_{\alpha}, x^{\prime}\right\rangle=\lim _{\alpha}\left\langle a e_{\alpha}, x^{\prime} y\right\rangle=\left\langle a, x^{\prime} y\right\rangle=\left\langle y a, x^{\prime}\right\rangle=\left\langle x, x^{\prime}\right\rangle,
$$

and so $x e_{\alpha} \stackrel{\text { weak }}{\longrightarrow} x$, as required.

For the converse, suppose that $x e_{\alpha} \stackrel{\text { weak }}{\longrightarrow} x$ in $X$ for every $x \in X$. Since $X \mathcal{A}$ is a closed subspace of $X$ (see for example [4, Section 2.9] or [17, Theorem 5.2.2]), this means that $x \in X \mathcal{A}$, as required.

The theorem above was proved in [14, Lemma 2.1] for $X=\mathcal{A}^{*}$. As a corollary, Lau and Ülger proved that $\mathcal{A}^{*}$ factors $\mathcal{A}$ if and only if $\mathcal{A}^{* *}$ has an identity. When $X=\mathcal{A}^{* *}$, we have the following.

Corollary 4.2. Let $\mathcal{A}$ be a Banach algebra $\mathcal{A}$ and $\left(e_{\alpha}\right)$ be a bai in $\mathcal{A}$ with a weak $k^{*}$ cluster point $e^{\prime \prime}$ in $\mathcal{A}^{* *}$. If $e_{\alpha} \stackrel{\text { weak }}{\longrightarrow} e^{\prime \prime}$ in $\mathcal{A}^{* *}$, then $\mathcal{A}^{* *}$ factors $\mathcal{A}$ on the left. If $e^{\prime \prime}$ is also a left identity (for instance when $\mathcal{A}$ is Arens regular), then the converse also holds.

Proof. The necessity follows directly from Theorem 4.5. In fact, $a^{\prime \prime} e_{\alpha}$ $\stackrel{\text { weak }}{\longrightarrow} a^{\prime \prime}$ since the map $b^{\prime \prime} \mapsto a^{\prime \prime} b^{\prime \prime}: \mathcal{A}^{* *} \rightarrow \mathcal{A}^{* *}$ is weak-weak continuous and $e^{\prime \prime}$ is a right identity in $\mathcal{A}^{* *}$. 
For the second statement, suppose that $e^{\prime \prime}$ is a left identity for $\mathcal{A}^{* *}$. Then again by Theorem $4.5, e_{\alpha}=e^{\prime \prime} e_{\alpha} \stackrel{\text { weak }}{\longrightarrow} e^{\prime \prime}$ in $\mathcal{A}^{* *}$.

Corollary 4.3. Let the Banach algebra $\mathcal{A}$ be a right ideal in $\mathcal{A}^{* *}$ and let $\left(e_{\alpha}\right)$ be a bai in $\mathcal{A}$ with a weak $k^{*}$ cluster point $e^{\prime \prime}$ in $\mathcal{A}^{* *}$. Then $e_{\alpha} \stackrel{\text { weak }}{\longrightarrow} e^{\prime \prime}$ in $\mathcal{A}^{* *}$ if and only if $\mathcal{A}^{* *}$ factors $\mathcal{A}$ on the left.

Proof. This follows from Theorem 4.2 and the corollary above.

\section{Topological centres of module extensions of Banach algebras.}

Let $\mathcal{A}$ be a Banach algebra and $X$ be a Banach $\mathcal{A}$-bimodule with module actions $\pi_{r}$ and $\pi_{l}$. Let $\mathcal{B}=X \oplus_{1} \mathcal{A}$ as a Banach space so that

$$
\|(x, a)\|=\|x\|+\|a\| \quad(a \in \mathcal{A}, x \in X) .
$$

Then $\mathcal{B}$ is a Banach algebra with the product

$$
(x, a)(y, b)=(x b+a y, a b) .
$$

The second dual $\mathcal{B}^{* *}$ of $\mathcal{B}$ is identified with $X^{* *} \oplus_{1} \mathcal{A}^{* *}$ as a Banach space, and the first Arens product on $\mathcal{B}^{* *}$ is given by

$$
\left(x^{\prime \prime}, a^{\prime \prime}\right)\left(y^{\prime \prime}, b^{\prime \prime}\right)=\left(x^{\prime \prime} b^{\prime \prime}+a^{\prime \prime} y^{\prime \prime}, a^{\prime \prime} b^{\prime \prime}\right),
$$

where $a^{\prime \prime} b^{\prime \prime}$ is as usual the first Arens product of $a^{\prime \prime}$ and $b^{\prime \prime}$ in $\mathcal{A}^{* *}, x^{\prime \prime} a^{\prime \prime}=$ $\pi_{r}^{* * *}\left(x^{\prime \prime}, a^{\prime \prime}\right)$ and $a^{\prime \prime} x^{\prime \prime}=\pi_{l}^{* * *}\left(a^{\prime \prime}, x^{\prime \prime}\right)$ for every $x^{\prime \prime} \in X^{* *}$ and $a^{\prime \prime} \in A^{* *}$. First, we determine the first topological centre of $\mathcal{B}^{* *}$. As in [12] or [6], we see from (1) that $\left(x^{\prime \prime}, a^{\prime \prime}\right) \in Z\left(\mathcal{B}^{* *}\right)$ if and only if

(i) $b^{\prime \prime} \mapsto a^{\prime \prime} b^{\prime \prime}: \mathcal{A}^{* *} \rightarrow \mathcal{A}^{* *}$ is weak ${ }^{*}$-weak ${ }^{*}$ continuous,

(ii) $y^{\prime \prime} \mapsto a^{\prime \prime} y^{\prime \prime}: X^{* *} \rightarrow X^{* *}$ is weak*-weak* continuous,

(iii) $b^{\prime \prime} \mapsto x^{\prime \prime} b^{\prime \prime}: \mathcal{A}^{* *} \rightarrow X^{* *}$ is weak*-weak* continuous.

Therefore we have

$$
Z\left(\mathcal{B}^{* *}\right)=Z_{\mathcal{A}}\left(X^{* *}\right) \times\left(Z_{X}\left(\mathcal{A}^{* *}\right) \cap Z\left(\mathcal{A}^{* *}\right)\right) .
$$

Secondly, we determine the second topological centre of $\mathcal{B}^{* *}$. The second Arens product of $\mathcal{B}^{* *}$ is given by

$$
\left(x^{\prime \prime}, a^{\prime \prime}\right) \cdot\left(y^{\prime \prime}, b^{\prime \prime}\right)=\left(x^{\prime \prime} \cdot b^{\prime \prime}+a^{\prime \prime} \cdot y^{\prime \prime}, a^{\prime \prime} \cdot b^{\prime \prime}\right),
$$

where $a^{\prime \prime} \cdot b^{\prime \prime}$ is as usual the second Arens product of $a^{\prime \prime}$ and $b^{\prime \prime}$ in $\mathcal{A}^{* *}$, $x^{\prime \prime} \cdot a^{\prime \prime}=\pi_{r}^{t * * * t}\left(x^{\prime \prime}, a^{\prime \prime}\right)$ and $a^{\prime \prime} \cdot x^{\prime \prime}=\pi_{l}^{t * * * t}\left(a^{\prime \prime}, x^{\prime \prime}\right)$ for every $x^{\prime \prime} \in X^{* *}$ and $a^{\prime \prime} \in A^{* *}$. Accordingly, $\left(y^{\prime \prime}, b^{\prime \prime}\right) \in Z^{t}\left(\mathcal{B}^{* *}\right)$ if and only if

(i) $a^{\prime \prime} \mapsto a^{\prime \prime} \cdot b^{\prime \prime}: \mathcal{A}^{* *} \rightarrow \mathcal{A}^{* *}$ is weak ${ }^{*}$-weak* continuous,

(ii) $x^{\prime \prime} \mapsto x^{\prime \prime} . b^{\prime \prime}: X^{* *} \rightarrow X^{* *}$ is weak ${ }^{*}$-weak ${ }^{*}$ continuous,

(iii) $a^{\prime \prime} \mapsto a^{\prime \prime} . y^{\prime \prime}: \mathcal{A}^{* *} \rightarrow X^{* *}$ is weak*-weak ${ }^{*}$ continuous.

Therefore we have

$$
Z^{t}\left(\mathcal{B}^{* *}\right)=Z_{\mathcal{A}}^{t}\left(X^{* *}\right) \times\left(Z_{X}^{t}\left(\mathcal{A}^{* *}\right) \cap Z^{t}\left(\mathcal{A}^{* *}\right)\right) .
$$


Now we are able to give a class of Banach algebras which are not strongly Arens irregular but are left strongly Arens irregular.

Theorem 5.1. Let $\mathcal{A}$ be a strongly irregular Banach algebra and let $X$ be a non-reflexive right $\mathcal{A}$-module with a module action $\pi_{r}: X \times \mathcal{A} \rightarrow X$ such that $\pi_{r}^{*}\left(x^{\prime}, X\right)=\mathcal{A}^{*}$ and $\pi_{r}^{* *}\left(\mathcal{A}^{* *}, x^{\prime}\right)=X^{*}$ for some $x^{\prime} \in X$ (these are the conditions imposed on $X$ in Theorem 2.2). Take the left module action of $\mathcal{A}$ on $X$ to be trivial. Then $\mathcal{B}=X \oplus_{1} \mathcal{A}$ is left strongly Arens irregular, but not right strongly Arens irregular.

Proof. By Theorem 2.2, we have $Z_{\mathcal{A}}\left(X^{* *}\right)=X$. Then, by (2), we have

$$
Z\left(\mathcal{B}^{* *}\right)=X \times\left(Z_{X}\left(\mathcal{A}^{* *}\right) \cap \mathcal{A}\right)=X \times \mathcal{A}=\mathcal{B} .
$$

In other words, $\mathcal{B}$ is left strongly Arens irregular. On the other hand, since the left module action of $\mathcal{A}$ on $X$ is trivial, $Z_{\mathcal{A}}^{t}\left(X^{* *}\right)=X^{* *}$. Then, by (4),

$$
Z^{t}\left(\mathcal{B}^{* *}\right)=X^{* *} \times\left(Z_{X}^{t}\left(\mathcal{A}^{* *}\right) \cap \mathcal{A}\right)=X^{* *} \times \mathcal{A} \neq \mathcal{B},
$$

which means that $\mathcal{B}$ is not right strongly Arens irregular.

REMARK. The careful reader would notice at this stage that if $\mathcal{A}$ is reflexive instead of strongly Arens irregular in Theorem 5.1, then $Z^{t}\left(\mathcal{B}^{* *}\right)=$ $X^{* *} \times \mathcal{A}=\mathcal{B}^{* *}$, and so $\mathcal{B}$ is Arens regular while $Z\left(\mathcal{B}^{* *}\right)=X \times \mathcal{A}=\mathcal{B}$. This is of course absurd unless $X$ is also reflexive. Indeed, the proposition below shows that this is the case.

Proposition 5.1. Let $\mathcal{A}$ and $X$ be as in Theorem 2.2, i.e., $X$ is a right $\mathcal{A}$-module with module action $\pi_{r}: X \times \mathcal{A} \rightarrow X$ and $x^{\prime} \in X^{*}$ such that $\pi_{r}^{*}\left(x^{\prime}, X\right)=\mathcal{A}^{*}$ and $\pi_{r}^{* *}\left(\mathcal{A}^{* *}, x^{\prime}\right)=X^{*}$. If $\mathcal{A}$ is reflexive, then $X$ is reflexive.

Proof. We show that the weak* and weak topologies coincide on $X^{*}$. So let $y_{\alpha}^{\prime} \stackrel{\text { weak }^{*}}{\longrightarrow} y^{\prime}$ in $X^{*}$. By hypothesis, we write $y_{\alpha}^{\prime}=\pi_{r}^{* *}\left(a_{\alpha}, x^{\prime}\right)$ and $y^{\prime}=$ $\pi_{r}^{* *}\left(a, x^{\prime}\right)$ for some net $\left(a_{\alpha}\right)$ in $\mathcal{A}, a \in \mathcal{A}$ and $x^{\prime} \in X^{*}\left(\right.$ since $\left.\mathcal{A}^{* *}=\mathcal{A}\right)$. We verify that in fact $a$ is the weak limit of $\left(a_{\alpha}\right)$ in $\mathcal{A}$. So let $a^{\prime} \in \mathcal{A}^{*}$ and write $a^{\prime}=\pi_{r}^{*}\left(x^{\prime}, x\right)$ for some $x \in X$. Then

$$
\begin{aligned}
\left\langle a_{\alpha}, a^{\prime}\right\rangle & =\left\langle a_{\alpha}, \pi_{r}^{*}\left(x^{\prime}, x\right)\right\rangle=\left\langle\pi_{r}^{* *}\left(a_{\alpha}, x^{\prime}\right), x\right\rangle=\left\langle y_{\alpha}^{\prime}, x\right\rangle \\
& \stackrel{\text { weak }^{*}}{\longrightarrow}\left\langle y^{\prime}, x\right\rangle=\left\langle\pi_{r}^{* *}\left(a, x^{\prime}\right), x\right\rangle=\left\langle a, \pi_{r}^{*}\left(x^{\prime}, x\right)\right\rangle=\left\langle a, a^{\prime}\right\rangle,
\end{aligned}
$$

as required. We then apply [8, Theorem V.3.15] to see that the map $a \mapsto$ $\pi_{r}^{* *}\left(a, x^{\prime}\right): \mathcal{A} \rightarrow X^{*}$ is weak-weak continuous, and accordingly, $y_{\alpha}^{\prime}=$ $\pi_{r}^{* *}\left(a_{\alpha}, x^{\prime}\right) \stackrel{\text { weak }}{\longrightarrow} \pi_{r}^{* *}\left(a, x^{\prime}\right)=y^{\prime}$ in $X^{*}$.

Corollary 5.1. Let $G$ be an infinite locally compact group. Consider $L^{\infty}(G)$ as an $L^{1}(G)$-module with the natural right module action and the trivial left module action. Then $L^{\infty}(G) \oplus_{1} L^{1}(G)$ is left strongly Arens irregular, but not right strongly Arens irregular. 
EXAmPLE 5.1. The construction above enables us also to give simple examples of Banach algebras which are neither Arens regular nor strongly Arens irregular. Let $\mathcal{A}$ be a Banach algebra as in Example 2.1. Then $Z_{\mathcal{A}^{*}}\left(\mathcal{A}^{* *}\right)=Z_{\mathcal{A}}\left(\mathcal{A}^{* *}\right)=\mathcal{A}^{* *}$ and $Z_{\mathcal{A}}\left(\mathcal{A}^{* * *}\right) \neq \mathcal{A}^{* * *}$. For $\mathcal{B}=\mathcal{A}^{*} \oplus_{1} \mathcal{A}$, we have $Z\left(\mathcal{B}^{* *}\right)=Z_{\mathcal{A}}\left(\mathcal{A}^{* * *}\right) \times \mathcal{A}^{* *}$. Therefore $\mathcal{B}^{* *} \neq Z\left(\mathcal{B}^{* *}\right) \neq \mathcal{B}$, showing that $\mathcal{B}$ is neither Arens regular nor strongly Arens irregular.

Another example of such an algebra has been given by Saghafi in [18], where she starts with a compact totally ordered space $X$ which is a semigroup under the multiplication $x y=\max \{x, y\}$, takes a continuous regular Borel measure $\mu$ on $X$ with $\operatorname{supp} \mu=X$ and considers the commutative Banach algebra $L^{1}(\mu)$ with convolution as multiplication, and proves that the algebraic centre (which agrees with the topological centre in this case) of $L^{1}(\mu)^{* *}$ equipped with the first Arens product is not the whole of $L^{1}(\mu)^{* *}$, but is much larger than $L^{1}(\mu)$.

6. Topological centres of triangular Banach algebras. In [11], Forrest and Marcoux found necessary and sufficient conditions for the Arens regularity of unital triangular Banach algebras. In this section, we find the topological centres of triangular Banach algebras and we use these centres to prove the following assertions:

(i) There exists a Banach algebra $\mathcal{T}$ such that $\mathcal{T}^{*}$ factors $\mathcal{T}$ only from one side and $Z\left(\mathcal{T}^{* *}\right)=Z^{t}\left(\mathcal{T}^{* *}\right)$. This shows that the existence of a bounded approximate identity in the algebra is necessary for the validity of [14, Proposition 2.10].

(ii) There is a Banach algebra $\mathcal{T}$ such that $\mathcal{T}^{*} \mathcal{T}=\mathcal{T} \mathcal{T}^{*}$ but $Z\left(\mathcal{T}^{* *}\right) \neq$ $Z^{t}\left(\mathcal{T}^{* *}\right)$. This answers negatively question $(6 \mathrm{~d})$ in [14].

(iii) There exists a Banach algebra $\mathcal{T}$ which is strongly Arens irregular, but it is not weakly sequentially complete. This answers negatively question $(6 \mathrm{j})$ in [14].

We should note that the Banach algebra in the list of questions given in [14] is throughout assumed to have a bai, while our $\mathcal{T}$ in (ii) and (iii) is even unital.

In [12], Ghahramani et al. also gave examples which are different from ours to answer these questions.

Let $\mathcal{A}$ and $\mathcal{B}$ be Banach algebras, and suppose that $X$ is a Banach $\mathcal{A}, \mathcal{B}$ module; that is, $X$ is a Banach space, a left $\mathcal{A}$-module and a right $\mathcal{B}$-module, and the action $\pi_{l}$ of $\mathcal{A}$ and the action $\pi_{r}$ of $\mathcal{B}$ are continuous, that is,

$$
\left\|\pi_{r}\left(\pi_{l}(a, x), b\right)\right\| \leq\|a\|_{\mathcal{A}}\|x\|_{X}\|b\|_{\mathcal{B}}
$$

for all $a \in \mathcal{A}, x \in X, b \in \mathcal{B}$. For simplicity, we shall write $\pi_{l}(a, x)$ as $a x$ and 
$\pi_{r}(x, b)$ as $x b$. We define the corresponding triangular Banach algebra by

$$
\mathcal{T}=\left(\begin{array}{cc}
\mathcal{A} & X \\
0 & \mathcal{B}
\end{array}\right)
$$

with the sum and product being given by the usual $2 \times 2$ matrix operations and obvious internal module actions. The norm on $\mathcal{T}$ is

$$
\left\|\left(\begin{array}{cc}
a & x \\
0 & b
\end{array}\right)\right\|:=\|a\|_{\mathcal{A}}+\|x\|_{X}+\|b\|_{\mathcal{B}}
$$

We can extend the actions of $\mathcal{A}$ and $\mathcal{B}$ on $X$ to actions of $\mathcal{A}^{* *}$ and $\mathcal{B}^{* *}$ on $X^{* *}$ via

$a^{\prime \prime} x^{\prime \prime}=\pi_{l}^{* * *}\left(a^{\prime \prime}, x^{\prime \prime}\right), x^{\prime \prime} b^{\prime \prime}=\pi_{r}^{* * *}\left(x^{\prime \prime}, b^{\prime \prime}\right) \quad$ for $x^{\prime \prime} \in X^{* *}, a^{\prime \prime} \in \mathcal{A}^{* *}, b^{\prime \prime} \in \mathcal{B}^{* *}$.

As in [11], the first Arens product of $\mathcal{T}^{* *}$ behaves just like matrix multiplication with coordinate-level operations behaving like the first Arens product of the building blocks. In other words, we have

$$
\left(\begin{array}{cc}
a_{1}^{\prime \prime} & x_{1}^{\prime \prime} \\
0 & b_{1}^{\prime \prime}
\end{array}\right)\left(\begin{array}{cc}
a_{2}^{\prime \prime} & x_{2}^{\prime \prime} \\
0 & b_{2}^{\prime \prime}
\end{array}\right)=\left(\begin{array}{cc}
a_{1}^{\prime \prime} a_{2}^{\prime \prime} & a_{1}^{\prime \prime} x_{2}^{\prime \prime}+x_{1}^{\prime \prime} b_{2}^{\prime \prime} \\
0 & b_{1}^{\prime \prime} b_{2}^{\prime \prime}
\end{array}\right) .
$$

Similarly for the second Arens product of $\mathcal{T}^{* *}$, we have

$$
\left(\begin{array}{cc}
a_{1}^{\prime \prime} & x_{1}^{\prime \prime} \\
0 & b_{1}^{\prime \prime}
\end{array}\right) \cdot\left(\begin{array}{cc}
a_{2}^{\prime \prime} & x_{2}^{\prime \prime} \\
0 & b_{2}^{\prime \prime}
\end{array}\right)=\left(\begin{array}{cc}
a_{1}^{\prime \prime} \cdot a_{2}^{\prime \prime} & a_{1}^{\prime \prime} \cdot x_{2}^{\prime \prime}+x_{1}^{\prime \prime} \cdot b_{2}^{\prime \prime} \\
0 & b_{1}^{\prime \prime} \cdot b_{2}^{\prime \prime}
\end{array}\right),
$$

where $a^{\prime \prime} \cdot x^{\prime \prime}=\pi_{l}^{t * * * t}\left(a^{\prime \prime}, x^{\prime \prime}\right)$ and $x^{\prime \prime} \cdot b^{\prime \prime}=\pi_{r}^{t * * * t}\left(x^{\prime \prime}, b^{\prime \prime}\right)$ for every $x^{\prime \prime} \in X^{* *}$, $a^{\prime \prime} \in \mathcal{A}^{* *}$ and $b^{\prime \prime} \in \mathcal{B}^{* *}$.

Applying (1) and (2), we obtain

TheOREM 6.1. Let $\mathcal{T}$ be as above. Then

$$
\begin{aligned}
Z\left(\mathcal{T}^{* *}\right) & =\left(\begin{array}{cc}
Z\left(\mathcal{A}^{* *}\right) \cap Z_{X}\left(\mathcal{A}^{* *}\right) & Z_{\mathcal{B}}\left(X^{* *}\right) \\
0 & Z\left(\mathcal{B}^{* *}\right)
\end{array}\right), \\
Z^{t}\left(\mathcal{T}^{* *}\right) & =\left(\begin{array}{cc}
Z^{t}\left(\mathcal{A}^{* *}\right) & Z_{\mathcal{A}}^{t}\left(X^{* *}\right) \\
0 & Z^{t}\left(\mathcal{B}^{* *}\right) \cap Z_{X}^{t}\left(\mathcal{B}^{* *}\right)
\end{array}\right) .
\end{aligned}
$$

Suppose now that $\mathcal{A}$ is a commutative unital Banach algebra, and let $\varphi$ be an element of the spectrum of $\mathcal{A}$. Then $\mathbb{C}$ is a Banach $\mathcal{A}$-bimodule with the following module actions:

$$
a c=c \varphi(a), \quad c a=0 \quad(a \in \mathcal{A}, c \in \mathbb{C}) .
$$

We denote $\mathbb{C}$ by $\mathbb{C}_{\varphi}$ as a Banach $\mathcal{A}$-bimodule. Since $\mathbb{C}$ is a reflexive Banach space, $Z_{\mathcal{B}}\left(\mathbb{C}_{\varphi}^{* *}\right)=Z_{\mathcal{A}}^{t}\left(\mathbb{C}_{\varphi}^{* *}\right)=\mathbb{C}_{\varphi}$. Let now $\mathcal{A}$ be a strongly Arens irregular 
Banach algebra (such as $l^{1}(\mathbb{Z})$ with convolution product) and let

$$
\mathcal{T}=\left(\begin{array}{cc}
\mathcal{A} & \mathbb{C}_{\varphi} \\
0 & \mathcal{A}
\end{array}\right)
$$

Then $Z\left(\mathcal{T}^{* *}\right)=Z^{t}\left(\mathcal{T}^{* *}\right)=\mathcal{T}$. On the other hand, $\mathcal{T}^{*}$ factors $\mathcal{T}$ from the left but does not factor from the right. Therefore we have the following corollary.

Corollary 6.1. There exists a Banach algebra $\mathcal{T}$ such that $\mathcal{T}^{*}$ factors $\mathcal{T}$ from one side only and $Z\left(\mathcal{T}^{* *}\right)=Z^{t}\left(\mathcal{T}^{* *}\right)$.

We now answer question $(6 \mathrm{~d})$ in [14].

Corollary 6.2. Let $\mathcal{A}$ be an infinite-dimensional unital $C^{*}$-algebra, $\mathcal{B}=\mathcal{A}$ and $X=\mathcal{A}^{*}$. Then $\mathcal{T}$ is unital, $\mathcal{T}^{*}$ factors $\mathcal{T}$ but $Z\left(\mathcal{T}^{* *}\right) \neq Z^{t}\left(\mathcal{T}^{* *}\right)$.

Proof. Since $\mathcal{A}$ is Arens regular, $Z\left(\mathcal{A}^{* *}\right)=Z^{t}\left(\mathcal{A}^{* *}\right)=\mathcal{A}^{* *}$. Let $\pi$ : $\mathcal{A} \times \mathcal{A} \rightarrow \mathcal{A}$ be the product of $\mathcal{A}$. Then by Corollary 2.3, the left module action of $\mathcal{A}$ on $\mathcal{A}^{*}$ is not Arens regular since $\mathcal{A}$ is of infinite dimension. This is equivalent to $Z_{X}\left(\mathcal{A}^{* *}\right) \neq \mathcal{A}^{* *}$. We see from Theorem 6.1 that

$$
Z\left(\mathcal{T}^{* *}\right)=\left(\begin{array}{cc}
Z_{X}\left(\mathcal{A}^{* *}\right) & Z_{\mathcal{A}}\left(X^{* *}\right) \\
0 & \mathcal{A}^{* *}
\end{array}\right), \quad Z^{t}\left(\mathcal{T}^{* *}\right)=\left(\begin{array}{cc}
\mathcal{A}^{* *} & Z_{\mathcal{A}}^{t}\left(X^{* *}\right) \\
0 & Z_{X}^{t}\left(\mathcal{A}^{* *}\right)
\end{array}\right) .
$$

Thus $Z\left(\mathcal{T}^{* *}\right) \neq Z^{t}\left(\mathcal{T}^{* *}\right)$. Clearly $\mathcal{T}^{*}$ factors $\mathcal{T}$ since $\mathcal{T}$ is unital with a unit element $\left(\begin{array}{ll}I & 0 \\ 0 & I\end{array}\right)$, where $I$ is the identity of $\mathcal{A}$.

We now prove assertion (iii). Let $\mathcal{A}=\mathcal{B}=l^{1}(\mathbb{Z})$ with convolution product, $X=\mathcal{A}^{*}$ and let $\mathcal{T}=\left(\begin{array}{cc}\mathcal{A} & X \\ 0 & \mathcal{B}\end{array}\right)$. Then $\mathcal{T}$ is unital, and it is easy to see that $Z_{\mathcal{A}}^{t}\left(X^{* *}\right)=Z_{\mathcal{B}}\left(X^{* *}\right)=\mathcal{A}^{*}$ since $\ell^{1}(\mathbb{Z})^{* *}$ has an identity. On the other hand, $\mathcal{A}$ is strongly Arens irregular; then by Theorem 6.1, $\mathcal{T}$ is strongly Arens irregular. It is easy to show that $\mathcal{T}$ is weakly sequentially complete if and only if $\mathcal{A}, \mathcal{B}$ and $X$ are weakly sequentially complete. Since we know that $X=l^{\infty}(\mathbb{Z})$ is not weakly sequentially complete, we have

COROLlary 6.3. There exists a unital strongly Arens irregular Banach algebra $\mathcal{T}$ which is not weakly sequentially complete.

Acknowledgments. We are indebted to the referee for the careful reading of the paper and the detailed criticism which greatly improved the presentation of the paper.

\section{References}

[1] R. Arens, Operations induced in function classes, Monatsh. Math. 55 (1951), 1-19.

[2] —, The adjoint of a bilinear operation, Proc. Amer. Math. Soc. 2 (1951), 839-848.

[3] N. Arıkan, Arens regularity and reflexivity, Quart. J. Math. Oxford Ser. 32 (1981), $383-388$. 
[4] H. G. Dales, Banach Algebras and Automatic Continuity, London Math. Soc. Monogr. 24, Clarendon Press, Oxford, 2000.

[5] H. G. Dales and A. T.-M. Lau, The second duals of Beurling algebras, Mem. Amer. Math. Soc. 177 (2005), no. 836.

[6] H. G. Dales, A. Rodríguez-Palacios and M. V. Velasco, The second transpose of a derivation, J. London Math. Soc. (2) 64 (2001), 707-721.

[7] J. Duncan and S. A. Hosseiniun, The second dual of a Banach algebra, Proc. Roy. Soc. Edinburgh Sect. A 84 (1979), 309-325.

[8] N. Dunford and J. Schwartz, Linear Operators I, Interscience, New York, 1966.

[9] M. Filali and P. Salmi, Slowly oscillating functions in semigroup compactifications and convolution algebras, J. Funct. Anal., to appear.

[10] M. Filali and A. I. Singh, Recent developments on Arens regularity and ideal structure of the second dual of a group algebra and some related toplogical algebras, in: General Topological Algebras (Tartu, 1999), Est. Math. Soc., 2001, 95-124.

[11] B. E. Forrest and L. W. Marcoux, Weak amenability of triangular Banach algebras, Trans. Amer. Math. Soc. 354 (2001), 1435-1452.

[12] F. Ghahramani, J. P. McClure and M. Meng, On asymmetry of topological centers of the second duals of Banach algebras, Proc. Amer. Math. Soc. 126 (1998), 1765-1768.

[13] A. T.-M. Lau and V. Losert, On the second conjugate of $L^{1}(G)$ of a locally compact group, J. London Math. Soc. 37 (1988), 464-470.

[14] A. T.-M. Lau and A. Ülger, Topological centers of certain dual algebras, Trans. Amer. Math. Soc. 348 (1996), 1191-1212.

[15] M. Neufang, A unified approach to the topological centre problem for certain Banach algebras arising in abstract harmonic analysis, Arch. Math. (Basel) 82 (2004), 164171.

[16] -, A quantized analogue of the convolution algebra $L_{1}(G)$, preprint, 2004.

[17] T. W. Palmer, Banach Algebras and the General Theory of *-Algebras, Vol. 1, Cambridge Univ. Press, 1994.

[18] A. Saghafi, On the $L^{1}$-algebras of some compact totally ordered spaces, Math. Proc. Cambridge Philos. Soc. 122 (1997), 173-184.

[19] A. Ülger, Weakly compact bilinear forms and Arens regularity, Proc. Amer. Math. Soc. 101 (1987), 697-704.

[20] - , Arens regularity sometimes implies the RNP, Pacific J. Math. 143 (1990), 377399.

[21] N. J. Young, The irregularity of multiplication in group algebras, Quart. J. Math. Oxford 24 (1973), 59-62.

Department of Mathematics

University of Semnan

Semnan, Iran

and

Department of Mathematics

Shahid Beheshti University

Tehran, Iran

E-mail: maj_ess@yahoo.com
Department of Mathematical Sciences University of Oulu Oulu 90014, Finland E-mail: mfilali@cc.oulu.fi

Received March 2, 2006

Revised version June 11, 2007 